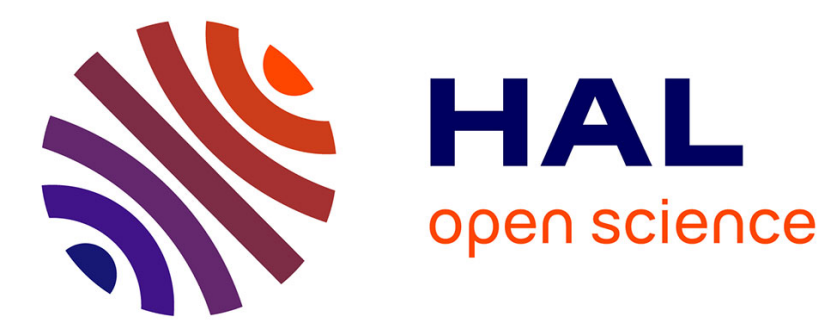

\title{
Critères diagnostics des uvéites tuberculeuses
}

Sarah Lechtman

\section{To cite this version:}

Sarah Lechtman. Critères diagnostics des uvéites tuberculeuses. Médecine humaine et pathologie.

2015. dumas-01293778

\section{HAL Id: dumas-01293778 \\ https://dumas.ccsd.cnrs.fr/dumas-01293778}

Submitted on 25 Mar 2016

HAL is a multi-disciplinary open access archive for the deposit and dissemination of scientific research documents, whether they are published or not. The documents may come from teaching and research institutions in France or abroad, or from public or private research centers.
L'archive ouverte pluridisciplinaire HAL, est destinée au dépôt et à la diffusion de documents scientifiques de niveau recherche, publiés ou non, émanant des établissements d'enseignement et de recherche français ou étrangers, des laboratoires publics ou privés. 


\title{
CRITERES DIAGNOSTICS DES UVEITES TUBERCULEUSES
} THESE PRESENTEE POUR L'OBTENTION DU DIPLOME D'ETAT DE DOCTEUR EN MEDECINE

\author{
Par Sarah LECHTMAN
}

19/05/2015

Présentée et soutenue publiquement à la Faculté de Médecine de Nice le 19/05/2015

Devant le Jury composé de :

Pr Fuzibet ; Président du Jury, Chef de service de Médecine Interne, hôpital L'Archet 1, CHU de Nice Pr Gastaud ; Chef de service d'Ophtalmologie, hôpital Saint-Roch, CHU de Nice Pr Lacour ; Chef de service de Dermatologie, hôpital L'Archet 2, CHU de Nice Dr Tieulié ; Directrice de thèse, service de médecine interne, hôpital L'Archet 1, Nice 


\section{UNIVERSITÉ DE NICE-SOPHIA ANTIPOLIS}

\section{FACULTÉ DE MÉDECINE}

Liste des professeurs au 1er septembre 2014 à la Faculté de Médecine de Nice

\begin{abstract}
Doyen
Assesseurs

Conservateur de la bibliothèque

Directrice administrative des services

Doyens Honoraires
\end{abstract}

\section{Professeurs Honoraires}

M. BALAS Daniel

M. BLAIVE Bruno

M. BOQUET Patrice

M. BOURGEON André

M. BOUTTÉ Patrick

M. BRUNETON Jean-Noël

Mme BUSSIERE Françoise

M. CAMOUS Jean-Pierre

M. CHATEL Marcel

M. COUSSEMENT Alain

M. DARCOURT Guy

M. DELLAMONICA Pierre

M. DELMONT Jean

M. DEMARD François

M. DOLISI Claude

M . FRANCO Alain

M. FREYCHET Pierre

M. GÉRARD Jean-Pierre

M. GILLET Jean-Yves

M. GRELLIER Patrick

M.C.A. Honoraire
M. BAQUÉ Patrick

M. ESNAULT Vincent

M. CARLES Michel

Mme BREUIL Véronique

Mme DE LEMOS Annelyse

Mme CALLEA Isabelle

M. AYRAUD Noël

M. RAMPAL Patrick

M. BENCHIMOL Daniel

M. HARTER Michel

M. INGLESAKIS Jean-André

M. LALANNE Claude-Michel

M. LAMBERT Jean-Claude

M. LAZDUNSKI Michel

M. LEFEBVRE Jean-Claude

M. LE BAS Pierre

M. LE FICHOUX Yves

M. LOUBIERE Robert

M. MARIANI Roger

M. MASSEYEFF René

M. MATTEI Mathieu

M. MOUIEL Jean

Mme MYQUEL Martine

M. OLLIER Amédée

M. ORTONNE Jean-Paul

M. SCHNEIDER Maurice

M. SERRES Jean-Jacques

M. TOUBOL Jacques

M. TRAN Dinh Khiem

M. ZIEGLER Gérard

Mlle ALLINE Madeleine 
M.C.U. Honoraires

M. ARNOLD Jacques

M. BASTERIS Bernard

Mlle CHICHMANIAN Rose-Marie

Mme DONZEAU Michèle

M. EMILIOZZI Roméo

M. FRANKEN Philippe

M. GASTAUD Marcel

\section{PROFESSEURS CLASSE \\ EXCEPTIONNELLE}

M. AMIEL Jean Urologie (52.04)

M. BENCHIMOL Daniel Chirurgie Générale (53.02)

M. BOILEAU Pascal Chirurgie Orthopédique et

Traumatologique (50.02)

M. DARCOURT Jacques Biophysique et

Médecine Nucléaire (43.01)

M. DESNUELLE Claude Biologie Cellulaire (44.03)

Mme EULLER-ZIEGLER Liana Rhumatologie (50.01)

M. FENICHEL Patrick Biologie du

Développement et de la Reproduction (54.05)

M. FUZIBET Jean-Gabriel Médecine Interne

(53.01)

M. GASTAUD Pierre Ophtalmologie (55.02)

M. GILSON Éric Biologie Cellulaire (44.03)

M. GRIMAUD Dominique Anesthésiologie et

Réanimation Chirurgicale (48.01)

\section{PROFESSEURS PREMIERE CLASSE}

M. BAQUÉ Patrick Anatomie - Chirurgie Générale (42.01)

M. BATT Michel Chirurgie Vasculaire (51.04)

M. BÉRARD Étienne Pédiatrie (54.01)

M. BERNARDIN Gilles Réanimation Médicale (48.02)

M. BONGAIN André Gynécologie-Obstétrique (54.03)

M. CASTILLO Laurent O.R.L. (55.01)

Mme CRENESSE Dominique Physiologie (44.02)
M.GIRARD-PIPAU Fernand

M. GIUDICELLI Jean

M. MAGNÉ Jacques

Mme MEMRAN Nadine

M. MENGUAL Raymond

M. POIRÉE Jean-Claude

Mme ROURE Marie-Claire

M. HASSEN KHODJA Reda Chirurgie

Vasculaire (51.04)

M. HÉBUTERNE Xavier Nutrition (44.04)

M. HOFMAN Paul Anatomie et Cytologie

Pathologiques (42.03)

M. LACOUR Jean-Philippe Dermato-

Vénéréologie (50.03)

Mme LEBRETON Élisabeth Chirurgie Plastique,

Reconstructrice et Esthétique (50.04)

M. MICHIELS Jean-François Anatomie et

Cytologie Pathologiques (42.03)

M. MOUROUX Jérôme Chirurgie Thoracique et

Cardiovasculaire (51.03)

M. PAQUIS Philippe Neurochirurgie (49.02)

M. PRINGUEY Dominique Psychiatrie

d'Adultes (49.03)

M. QUATREHOMME Gérald Médecine Légale

et Droit de la Santé (46.03)

M. M.ROBERT Philippe Psychiatrie d'Adultes

(49.03)

M. SANTINI Joseph O.R.L. (55.01)

M. THYSS Antoine Cancérologie, Radiothérapie (47.02)

M. VAN OBBERGHEN Emmanuel Biochimie et Biologie Moléculaire (44.01)

M. DE PERETTI Fernand Anatomie-Chirurgie Orthopédique (42.01)

M. DRICI Milou-Daniel Pharmacologie Clinique (48.03)

M. ESNAULT Vincent Néphrologie (52-03)

M. FERRARI Émile Cardiologie (51.02)

M. GIBELIN Pierre Cardiologie (51.02)

M. GUGENHEIM Jean Chirurgie Digestive (52.02)

Mme ICHAI Carole Anesthésiologie et

Réanimation Chirurgicale (48.01) 
M. LONJON Michel Neurochirurgie (49.02)

M. MARQUETTE Charles-Hugo Pneumologie (51.01)

M. MARTY Pierre Parasitologie et Mycologie (45.02)

M. MOUNIER Nicolas Cancérologie,

Radiothérapie (47.02)

M. PADOVANI Bernard Radiologie et Imagerie Médicale (43.02)

Mme PAQUIS Véronique Génétique (47.04)

\section{PROFESSEURS DEUXIEME CLASSE}

M. ALBERTINI Marc Pédiatrie (54.01) Mme ASKENAZY-GITTARD Florence Pédopsychiatrie (49.04)

M. BAHADORAN Philippe Cytologie et Histologie (42.02)

M. BARRANGER Emmanuel Gynécologie Obstétrique (54.03)

M. BENIZRI Emmanuel Chirurgie Générale (53.02)

Mme BLANC-PEDEUTOUR Florence

Cancérologie - Génétique (47.02)

M. BREAUD Jean Chirurgie Infantile (54-02)

Mlle BREUIL Véronique Rhumatologie (50.01)

M. CANIVET Bertrand Médecine Interne

(53.01)

M. CARLES Michel Anesthésiologie

Réanimation (48.01)

M. CASSUTO Jill-Patrice Hématologie et

Transfusion (47.01)

M. CHEVALLIER Patrick Radiologie et

Imagerie Médicale (43.02)

Mme CHINETTI Giulia Biochimie-Biologie

Moléculaire (44.01)

M. DUMONTIER Christian Chirurgie plastique

M. FERRERO Jean-Marc Cancérologie ;

Radiothérapie (47.02)
M. RAUCOULES-AIMÉ Marc Anesthésie et

Réanimation Chirurgicale (48.01)

Mme RAYNAUD Dominique Hématologie (47.01)

M. ROSENTHAL Éric Médecine Interne (53.01)

M. SCHNEIDER Stéphane Nutrition (44.04)

M. THOMAS Pierre Neurologie (49.01)

M. TRAN Albert Hépato Gastro-entérologie (52.01)

M. FONTAINE Denys Neurochirurgie (49.02)

M. FOURNIER Jean-Paul Thérapeutique (48-04)

M. FREDENRICH Alexandre Endocrinologie,

Diabète et Maladies métaboliques (54.04)

Mlle GIORDANENGO Valérie Bactériologie-

Virologie (45.01)

M. GUÉRIN Olivier Gériatrie (48.04)

M. HANNOUN-LEVI Jean-Michel Cancérologie ; Radiothérapie (47.02)

M. IANNELLI Antonio Chirurgie Digestive (52.02)

M. JOURDAN Jacques Chirurgie Thoracique et

Cardiovasculaire (51.03)

M. LEVRAUT Jacques Anesthésiologie et

Réanimation Chirurgicale (48.01)

M. PASSERON Thierry Dermato-Vénéréologie (50-03)

M. PICHE Thierry Gastro-entérologie (52.01)

M. PRADIER Christian Épidémiologie,

Économie de la Santé et Prévention (46.01)

M. ROGER Pierre-Marie Maladies Infectieuses ;

Maladies Tropicales (45.03)

M. ROHRLICH Pierre Pédiatrie (54.01)

M. RUIMY Raymond Bactériologie-virologie (45.01)

Mme SACCONI Sabrina Neurologie (49.01)

M. SADOUL Jean-Louis Endocrinologie,

Diabète et Maladies Métaboliques (54.04)

M. STACCINI Pascal Biostatistiques et

Informatique Médicale (46.04)

M. TROJANI Christophe Chirurgie

Orthopédique et Traumatologique (50.02)

M. VENISSAC Nicolas Chirurgie Thoracique et

Cardiovasculaire (51.03) 


\section{PROFESSEUR DES UNIVERSITÉS}

M. SAUTRON Jean-Baptiste Médecine Générale

\section{MAITRES DE CONFÉRENCES DES UNIVERSITÉS - PRATICIENS HOSPITALIERS}

Mme ALUNNI Véronique Médecine Légale et Droit de la Santé (46.03)

M. AMBROSETTI Damien Cytologie et

Histologie (42.02)

Mme BANNWARTH Sylvie Génétique (47.04)

M. BENOLIEL José Biophysique et Médecine

Nucléaire (43.01)

Mme BERNARD-POMIER Ghislaine

Immunologie (47.03)

Mme BUREL-VANDENBOS Fanny Anatomie

et Cytologie pathologiques (42.03)

M. DELOTTE Jérôme Gynécologie-Obstétrique

(54.03)

M. DOGLIO Alain Bactériologie-Virologie (45.01)

M. FOSSE Thierry Bactériologie-Virologie-

Hygiène (45.01)

M. GARRAFFO Rodolphe Pharmacologie

Fondamentale (48.03)

Mme GIOVANNINI-CHAMI Lisa Pédiatrie (54.01)

\section{PROFESSEURS ASSOCIÉS}

M. HOFLIGER Philippe Médecine Générale Mme POURRAT Isabelle Médecine Générale M. PRENTKI Marc Biochimie et Biologie moléculaire

\section{MAITRES DE CONFÉRENCES ASSOCIÉS}

Mme CHATTI Kaouthar Biophysique et Médecine Nucléaire

M. DARMON David Médecine Générale

MI. GARDON Gilles Médecine Générale

Mme MONNIER Brigitte Médecine Générale

M. PAPA Michel Médecine Générale
Mme HINAULT Charlotte Biochimie et biologie moléculaire (44.01)

Mlle LANDRAUD Luce Bactériologie-

Virologie (45.01)

Mme LEGROS Laurence Hématologie et

Transfusion (47.01)

Mme MAGNIÉ Marie-Noëlle Physiologie (44.02)

Mme MOCERI Pamela Cardiologie (51.02)

Mme MUSSO-LASSALLE Sandra Anatomie et Cytologie pathologiques (42.03)

M. NAÏMI Mourad Biochimie et Biologie moléculaire (44.01)

M. PHILIP Patrick Cytologie et Histologie (42.02)

Mme POMARES Christelle Parasitologie et mycologie (45.02)

M. ROUX Christian Rhumatologie (50.01)

M. TESTA Jean Épidémiologie Économie de la Santé et Prévention (46.01)

M. TOULON Pierre Hématologie et Transfusion (47.01) 
A ma grand-mère, Hélène Schulman Rose, qui n'est malheureusement plus parmi nous, À qui je dédie cette thèse.

Je sais que tu l'attendais depuis le dernier jour où je t'ai vu et que tu serais fière.

C'est toi qui m'as guidée de là-haut, j'en suis sûre et qui a veillé à ce que j'arrive au bout. J'aurai tellement voulu que tu sois là. 


\section{Remerciements}

A mon Président du Jury de thèse,

Mr le Professeur Jean-Gabriel Fuzibet, Professeur de médecine interne : Je vous remercie de me faire l'honneur de présider ce jury de thèse. Merci de m'avoir accueillie dans votre service durant ces années d'internat et pour la confiance que vous m'avez accordée auprès des patients.

A mes Juges,

Mr le Professeur Pierre Gastaud, Professeur d'Ophtalmologie : Merci d'avoir accepté de juger ce travail. J'espère que vous le trouverez intéressant.

Mr le Professeur Jean-Philippe Lacour, Professeur de Dermatologie : Je vous remercie de participer à ce jury de thèse. Merci pour mon semestre riche en expérience dans votre service et pour votre implication dans la formation de vos internes.

A ma directrice de thèse,

Mme le Docteur Nathalie Tieulié, Praticien-Hospitalier en médecine interne : Merci pour ta patience pour ce travail, pour la confiance que tu m'as accordée en me le proposant. Merci pour ta motivation auprès des internes et des patients. Merci pour les cours privés du vendredi après-midi et pour ton rôle de guide sur ce long chemin de l'apprentissage.

A Mme le Docteur Stéphanie Baillif, Praticien-Hospitalier en ophtalmologie : merci pour vos conseils et votre aide précieuse dans la réalisation de ce travail.

A Mr Kevin Zorzi : Merci pour ton aide dans la réalisation des statistiques.

Je tiens également à remercier mes maîtres de stage d'internat :

Du service de Médecine interne à l'Archet : A Mrs les Professeurs Jean-Gabriel Fuzibet et

\section{Eric Rosenthal.}

Merci à tous, avec qui j'ai eu la chance de partager tant des moments médicaux que personnels, dans ce service qui est devenu une deuxième maison.

A Mme le Docteur Viviane Queyrel : merci pour ta pédagogie et ton aide tout au long de mon cursus, merci pour ce que j'ai appris avec toi : sur les maladies mais aussi sur et avec les patients.

A Mr le Docteur Pierre-Yves Jeandel : Merci pour les coups de «boosts », les conseils et la confiance que tu m'as accordé.

A Mr le Docteur Jean Castela : merci pour votre savoir-faire. Je me rends compte de la chance que nous avons de vous avoir dans le service, pour nous apprendre les gestes au lit du malade.

Du service de Diabétologie de Pasteur :

A Mr le Professeur Bertrand Canivet et à Mr le Docteur Julien Algava : merci de m'avoir accompagné pour ce premier semestre. 
Du service de Néphrologie de Pasteur :

A Mr le Professeur Vincent Esnault : merci pour votre pédagogie et la confiance que vous m'avez témoigné pendant mon semestre.

A Mme le Docteur Barbara Seitz-Polski : merci de m'avoir transmis l'amour de la néphrologie, de m'avoir aidé à grandir et grâce à qui je suis ressortie plus forte du service.

A Mrs les Docteurs Guillaume Favre et Olivier Moranne : Merci pour votre pédagogie.

Du service de médecine interne de Cannes :

A Mme le Docteur Nathalie Montagne : merci infiniment pour votre humour, votre gentillesse et votre enthousiasme dans votre travail. Je me souviendrai à jamais des visites en musique et de la bonne humeur que vous communiquez aux patients.

A Mme le Docteur Annick Boscagli, modèle professionnel et humain : merci de m'avoir fait découvrir les joies de notre belle spécialité.

A Mrs les Docteurs Régis Kaphan et Matteo Vassalo : vous êtes des médecins à qui on souhaite ressembler, impliqués, joyeux et toujours disponibles.

Du service de maladies infectieuses et tropicales de l'Archet :

A Mr le Professeur Pierre-Marie Roger, Mme le Docteur Francine De Salvador, Mme le Docteur Evelyne Bernard, Mme le Docteur Karine Risso et Mr le Docteur Eric Denis: merci pour votre apprentissage et votre rôle de compagnonnage avec les internes.

A Mr le Docteur Eric Cua : J'ai été ravie de travailler avec toi. Merci pour ton aide et ta disponibilité.

Du service de réanimation médicale de l'Archet :

A Mr le Professeur Gérard Bernardin : Merci pour votre implication dans le pôle, la volonté d'une médecine de meilleure qualité, et d'un hôpital digne de la confiance des patients.

A Mr le Professeur Jean Dellamonica : Je te remercie pour ta pédagogie, ta patience, ta disponibilité et ton écoute. Les patients, dans leur malheur, ont de la chance de t'avoir, un médecin qui impose le respect et dont la seule présence rassure les équipes.

A Mr le Docteur Hervé Hyvernat : Merci pour ta patience et ton calme. Ta sérénité est un grand enseignement.

A Mrs les Docteurs Denis Doyen et Rémi Ladzunski : merci pour votre humour et la spéciale formation baby-foot. Merci pour les cathés...

Du service de Dermatologie de l'Archet :

A Mr le Professeur Thierry Passeron : merci pour votre pédagogie et votre enthousiasme.

A Mme le Docteur Emeline Castela : merci pour les bons moments passés en stage avec toi et pour ta gentillesse. Promis, je ne me trompe plus dans les prescriptions des dermocorticoïdes. 
A Mr le Docteur Henri Montaudier : merci de m'avoir fait partager ton savoir-faire avec les patients, ton expérience dans l'annonce diagnostique et l'accompagnement des familles.

Du service de Médecine interne de Bichat, à Paris :

A Mr le Professeur Thomas Papo : Vous êtes un exemple, votre savoir est impressionnant. Je suis ravie d'avoir eu l'honneur de travailler avec vous.

A Mme le Docteur Marie-Paule Chauveheid : Merci pour ta rigueur, ta gentillesse, ton paternalisme envers tes patients mais aussi tes internes. Je suis sincèrement heureuse d'avoir eu la chance de travailler à tes côtés et j'aimerai suivre ton exemple.

A Mr le Professeur Karim Sacré : merci de m'avoir accordé ta confiance et pour ton enthousiasme avec tous.

Je vous remercie tous de m'avoir accueillie dans cette grande maison, où notre travail devient vraiment un art, une passion : A Mme le Docteur Thyphaine Goulenok, Mme le Docteur Delphine Larivière, Mr le Docteur Antoine Dossier, Mr le Docteur Jean-François Alexandra, Mr le Docteur Mario Boisseau, Mr le Docteur Romain Jouenne.

Du service de Médecine interne de Lariboisière, à Paris :

A Mr le Professeur Jean-François Bergmann, merci pour votre dévotion auprès des étudiants. C'est grâce à vous qu'est née ma vocation pour notre spécialité.

Je tiens à remercier bien sûr les équipes de chaque service où je suis passée, pour m'avoir accueillie, soutenue et avoir accepté de travailler avec moi pour le même objectif auprès des malades.

En particulier, j'embrasse :

Jessica et Aurore, mes infirmières d'amour! Qui m'ont vu passer par tous les moments difficiles heureux ou tristes de l'internat ! Et un grand merci aux 2 Sandrines qui illuminent le service.

Tania et l'équipe de choc de Cannes ! Une spéciale dédicace à Mélanie qui est partie à Besançon.

Magali de l'HDS à Paris !

Oliv', Hoai, Eme' et l'équipe du 4èB !

Pascal, Violaine, Tareq et l'équipe de choc du STC néphro !

Mélanie, Bianca et toutes les infirmières et AS de la réa méd!

J'attribue un énorme merci à mes co-internes de spécialité qui partagent avec moi les doutes et traversent ce même chemin semé d'embûches. Merci de rester motivés, ensemble on s'émule et on est plus forts : Johan, Véréna, Nihal, Astrid, David, Sonia (même si elle a quitté notre route), Margaux et Diane et les nouveaux que je ne connais pas encore. Eléa bien sûr, qui est notre modèle !

Je n'oublie pas non plus : Fanny, qui est repartie à Paris, Marine Berguignat, qui m'a beaucoup appris, Guillaume, mon partenaire de galère à Cannes, Cédric qui a changé la tournure des visites d'infectieux, Marine Lochouarn toujours de bonne humeur, Florence ma James bond girl;-) qui m'a supportée au pire moment...Léo, pour nos nocturnes dans le bureau de Domi, Alex' et son croco score, Coralie, grâce à toi, un beau semestre et bien sûr, les parisiens, en particulier Claire, Stéphanie, Charlotte et Arthur !

A mes amis, et en priorité à ceux avec qui j'ai fait un bout de route sur les bancs de la faculté : 
Elodie, avec qui on a tout traversé. On y est arrivé ! et à Dam' pour la Bretagne, le wei et la mise en page!

Maud, pour les premières années et le Sénégal.

Jeff, Noé, Damien, Jean-Baptiste et Elodie, Clem', Hassan, Lolo, amis et co-externes et tellement de souvenirs...

Puis sont venus Caro et Alice, sur le tard, mais toujours là !

L'équipe des internes survivants de l'internat de Pasteur! A Camille et PPD, pour m'avoir accueillie à Nice. A Laura et Arié, pour m'avoir ouvert leur foyer. A Céline, ma coloc' et ophtalmo de choc.

Et puis à mes amis de toujours, qui me connaissent depuis la $6^{\mathrm{e}}$ : Marie, Aurélia et Carlo. Et spéciale dédicace à Sarah, pour notre projet initial commun et qui doit être quelque part en Allemagne maintenant...

Et enfin et surtout à ma famille, sans qui je ne serai jamais arrivée jusque là.

Parce que sans vous, je ne serai pas debout, Merci de tout mon cœur, de toute mon âme,

José-Mario-Malka, mis padres, por creer en mi por lo que puedo recordar, Pour avoir veillé à ce que je ne sois jamais en retard

De la enfermera a la policia, de la medicina para humanitaria, vous avez toujours été là !

Nunca podré olbidar su devocion, esta escrito para siempre en mi corazon !

Eva, mi amiga, mi hermana, mi alegria, qu'aurais-je fait sans toi ?

Fous-rires ou soupirs, même dans le pire, c'étaient nos meilleurs délires.

Coca-cola ou fanta, si ce n'est pas toi qui y crôis, c'est moi

Avec ces souvenirs, my dear, je n'ai plus rien à t'offrir.

Benji, depuis 1998, top départ, tu m'as donné l'espoir !

L'année du ballon rond, tu es né pour gagner.

Et pour P1, bien sûr, tu n'y es pas pour rien.

Car réciter, y a que toi, qui pouvais supporter.

J'en suis bien grée, merci pour tout ce que tu m'as donné.

Papi, à toi aussi, je dis merci,

Car les études, c'est ton prélude,

Alors pour te venger, j'y suis arrivée.

I por finir a mis abuelos i tios de Costa Rica. 


\section{I - Introduction}

\section{Contexte:}

A l'heure actuelle, le pathogène Mycobacterium tuberculosis infecte un tiers de la population mondiale, mais ne cause une maladie que chez $10 \%$ de ces individus. La tuberculose reste un problème de santé publique et a même été déclarée par l'Organisation Mondiale de la Santé «problème de santé planétaire urgent » dès 1993 [6]. Pourtant lorsqu'elle est correctement diagnostiquée, elle peut être traitée efficacement.

Dans $90 \%$ des cas, le bacille tuberculeux reste à l'état quiescent et ne provoque pas de maladie, c'est la tuberculose latente. La découverte d'une uvéite dans ce contexte, doit être interprétée avec prudence, puisqu'elle entraîne une sanction thérapeutique lourde. La prise en charge de ces uvéites est toujours un sujet de controverse. Selon les études, la tuberculose oculaire représenterait $18 \%$ des patients si un examen ophtalmologique était réalisé de façon systématique. Ces patients ne représentent que $2 \%$ des uvéites mais $50 \%$ des uvéites idiopathiques [5].

L'atteinte oculaire résulterait de deux mécanismes physiopathologiques différents : soit d'une infection intra-oculaire par les mycobactéries (dissémination hématogène d'une tuberculose maladie), soit d'une réaction d'hypersensibilité de type IV (réactivation au cours d'une tuberculose latente extra-oculaire) [4].

Ceci explique la variabilité de la présentation clinique. Toutes les structures de l'œil peuvent être touchées, s'exprimant par des atteintes peu spécifiques (conjonctivite, kératite, sclérite) ou de façon plus spécifique par une uvéite volontiers granulomateuse antérieure et bilatérale, parfois une hyalite controlatérale, une choroïdite granulomateuse ou serpigineuse ou très rarement par des tubercules de Bouchut (sauf lorsque le Mycobacterium dissémine par voie hématogène) [13].Une névrite optique ou une vasculite rétinienne peuvent également s'y associer.

Le diagnostic formel des uvéites tuberculeuses pose de nombreuses difficultés, en dehors des rares cas de tuberculose maladie, ou l'on peut mettre en évidence l'ADN du Mycobactérium 
tuberculosis par PCR réalisée sur un prélèvement d'humeur acqueuse [23]. La plupart des patients développant des manifestations oculaires n'ont aucune histoire de tuberculose pulmonaire ou extra-pulmonaire et leur présentation clinique est aspécifique, faisant errer le diagnostic. Beaucoup d'études sur les uvéites tuberculeuses reposent sur la démonstration de l'association d'une tuberculose systémique active ou latente avec une uvéite granulomateuse et/ou compatible. Mais toutes ces études ont évalué des moyens diagnostics différents, et peu d'entre-elles cumulent les différentes techniques disponibles (terrain, présentation clinique, radiographie, scanner thoracique, intra-dermoréaction à la tuberculine, test Interféron-gammarelease assay (IGRA) ou prélèvement d'humeur acqueuse par ponction de chambre antérieure). Et même si Gupta et al. ont récemment proposé une nouvelle classification de la tuberculose oculaire, basée sur les signes cliniques, les diverses investigations systémiques et paracliniques et le test thérapeutique, ils n'ont pas mené d'étude comparative pour valider leurs résultats [11].

\section{Objectif de l'étude :}

Nous avons souhaité établir des critères diagnostics d'uvéite tuberculeuse permettant d'orienter la pratique clinique, à partir de la comparaison d'une cohorte d'uvéites tuberculeuses à d'autres uvéites granulomateuses non tuberculeuses (la sarcoïdose, représentant le principal diagnostic différentiel et la syphilis, de diagnostic plus évident, afin d'avoir une population témoin bien définie). 


\section{II - Matériels et Méthodes}

Schéma de l'étude : Nous avons réalisé une étude observationnelle descriptive rétrospective monocentrique de cas/témoins à partir de patients suivis conjointement par les services de médecine interne et d'ophtalmologie du Centre Hospitalo-Universitaire de Nice, pour une uvéite granulomateuse.

Les patients ayant une uvéite tuberculeuse, diagnostiquée au CHU de Nice entre 2004 et 2014 ont été inclus et comparés aux patients suivis pour une uvéite syphilitique ou sarcoïdosique.

Les uvéites syphilitiques étaient de diagnostic évident : il s'agissait de patients ayant une uvéite avec une sérologie syphilis positive au diagnostic et guérie secondairement après une antibiothérapie adaptée (pénicilline $\mathrm{G}$ ou céphalosporines de $3^{\mathrm{e}}$ génération une fois par semaine ou une fois par jour pendant trois semaines pour respectivement une syphilis secondaire ou une neuro-syphilis).

Les uvéites d'origine sarcoïdosique présentaient une clinique ophtalomologique compatible, un granulome épithélioïde sans nécrose caséeuse sur une biopsie et/ou un tableau évocateur de sarcoïdose, après exclusion d'autres diagnostics possibles. Parmi ces dernières, seules étaient inclues les uvéites sévères; les neuropathies optiques rétro-bulbaires et épisclérites ont été exclues. Celles-ci ont été traitées par corticoïdes seuls ou en association à un immunosuppresseur (méthotrexate ou cyclophosphamide).

Etaient considérées comme des uvéites tuberculeuses, selon les critères de Graham et al. [10], les uvéites granulomateuses suspectes de tuberculose après exclusion des autres diagnostics, ayant répondu favorablement au traitement d'épreuve puis d'entretien antituberculeux.

Le traitement consistait en :

- un traitement d'attaque par: une quadri antibiothérapie antituberculeuse (rifampycine, isoniazide, pyrazinamide, éthambutol) pour les tuberculoses maladies ou une triantibiothérapie (rifampycine, isoniazide, pyrazinamide) pour les tuberculoses latentes pendant au moins deux mois 
- puis un traitement d'entretien par une bi antibiothérapie (rifampycine, isoniazide) pendant quatre à dix mois selon les cas, parfois associée à une corticothérapie locale voire systémique après le traitement d'attaque.

Nous avons recueillis pour chaque patient :

- Des données épidémiologiques :

âge, sexe, date du diagnostic, origine ethnique, voyage dans des pays d'endémie tuberculeuse ou contage, antécédent de BCG, existence d'une séropositivité VIH.

- Les caractéristiques de la présentation ophtalmologique : latéralisation de l'uvéite, localisation antérieure, intermédiaire, postérieure ou panuvéite, le caractère récidivant, hypertensif de l'uvéite, la présence d'une choroïdite serpigineuse, de nodules de Bouchut et l'existence d'une cataracte secondaire (témoin d'une inflammation chronique de l'œil).

- La présentation systémique: l'existence de signes généraux (fièvre, asthénie, anorexie, amaigrissement) et extra-pulmonaires (rhumatologiques, neurologiques ou cutanés) et de signes pulmonaires.

- Le profil biologique (numération formule sanguine, ionogramme sanguin, bilan hépatique et électrophorèse des protéines plasmatiques), virologique (statut VIH, VHB, VHC, syphilis, toxoplasmose, HSV, rickettsie, toxocarose et bartonnellose), immunologique (enzyme de conversion de l'angiotensine, typage HLA)

- Les moyens habituels d'orientation diagnostique: intradermo-réaction, test IGRA (Quantiféron $^{\circ}$ )

- Les examens d'imagerie: radiographie standard ou scanner thoracique avec la présence ou non de signes évocateurs (adénopathies, calcifications, syndrome interstitiel, nodule ou excavation)

- La bactériologie si celle-ci avait été réalisée : recherche de Bacilles Acido-AlcooloRésistants dans les expectorations, le lavage broncho-alvéolaire, la ponction lombaire ou la ponction de chambre antérieure 
- L’histologie si réalisée : présence d'un granulome épithélioïde.

Les patients du groupe uvéites tuberculeuses (UT) ont ensuite été comparés à ceux du groupe uvéites syphilitiques (USy) et du groupe uvéites sarcoïdosiques (USa) concernant chaque donnée recueillie.

Analyse statistique: Chaque variable a été comparée entre les deux groupes (uvéites tuberculeuses UT versus uvéites non tuberculeuses UNT) et nous avons dégagé celles qui étaient significativement différentes afin d'établir des critères diagnostiques d'uvéite tuberculeuse.

Les variables ont été comparées en analyse univariée et multivariée avec le test du Chi2 et le test exact de Fisher. 


\section{III - Résultats}

\section{Caractéristiques des patients :}

11 uvéites syphilitiques, 13 uvéites sarcoïdosiques et 22 uvéites tuberculeuses ont été inclues dans l'étude, à partir de la liste de patients suivis dans les services de médecine interne et d'ophtalmologie du Centre Hospitalo-Universitaire de Nice. Tous avaient été diagnostiqués entre 2004 et 2014. (Tableau 1).

Le groupe uvéite syphilitique comportait 81\% (9/11) hommes, d'âge moyen 59 ans (44-79). $18 \%(2 / 11)$ étaient d'origine Maghrébine et 28\% (3/11) avaient voyagé en zone d'endémie tuberculeuse. 18\% (2/11) étaient séropositifs pour le VIH.

Le groupe uvéite sarcoïdosique comportait 61\% (8/13) femmes, d'âge moyen 40,5 ans (2358). $23 \%(3 / 13)$ étaient d'origine Maghrébine et $7 \%(1 / 13)$ Africaine et $38 \%(5 / 13)$ avaient voyagé en Asie du Sud Est. Aucun patient n'était séropositif pour le VIH.

Le groupe uvéite tuberculeuse comportait 54\% (12/22) hommes, d'âge moyen 57,9 ans (2291). $71 \%(15 / 21)$ étaient d'origine de pays d'endémie tuberculeuse et $90 \%(19 / 22)$ avaient voyagé en zone endémique. On notait un patient qui avait présenté une uvéite tuberculeuse suite à une BCG thérapie intra-vésicale et un patient séropositif pour le VIH. 


\begin{tabular}{|c|c|c|c|c|c|c|}
\hline $\mathrm{n}$ & âge & sexe & ethnie & Voyage en ZE & BCG & VIH \\
\hline UT & $\begin{array}{l}57,9 \\
(22-91)\end{array}$ & $12(54 \%) \mathrm{H}$ & $\begin{array}{l}15(71 \%) \text { origine } \mathrm{ZE} \\
8(36 \%) \text { Afrique } \\
5(23 \%) \text { Maghreb } \\
2(9 \%) \text { Asie }\end{array}$ & $19(90 \%)$ & $1(4 \%)$ & $1(4 \%)$ \\
\hline 1 & 85 & $\mathrm{~F}$ & Cap Vert & oui & ND & ND \\
\hline 2 & 50 & $\mathrm{H}$ & Mali & oui & non & oui \\
\hline 3 & 91 & $\mathrm{H}$ & France & non & Post-BCG intra-vésical & non \\
\hline 4 & 33 & $\mathrm{H}$ & Maroc & oui & ND & non \\
\hline 5 & 61 & $\mathrm{H}$ & Sénégal & oui & oui & non \\
\hline 6 & 86 & $\mathrm{H}$ & ND & ND & ND & non \\
\hline 7 & 77 & $\mathrm{H}$ & Maroc & oui & ND & non \\
\hline 8 & 80 & $\mathrm{H}$ & France & ND & ND & non \\
\hline 9 & 53 & $\mathrm{~F}$ & Maroc & oui & ND & non \\
\hline 10 & 47 & $\mathrm{~F}$ & Vietnam & oui & ND & non \\
\hline 11 & 85 & $\mathrm{~F}$ & Madagascar & oui & ND & non \\
\hline 12 & 41 & $\mathrm{~F}$ & Cameroun & oui & ND & non \\
\hline 13 & 55 & $\bar{F}$ & France & oui Madagascar & ND & non \\
\hline 14 & 33 & $\mathrm{H}$ & Sénégal & oui & ND & non \\
\hline 15 & 39 & $\mathrm{~F}$ & Inde & oui & ND & non \\
\hline 16 & 35 & $\mathrm{H}$ & Côte d'Ivoire & oui & ND & non \\
\hline 17 & 32 & $\mathrm{~F}$ & Burkina & oui & $\mathrm{ND}$ & non \\
\hline 18 & 87 & $\mathrm{~F}$ & France & oui & ND & non \\
\hline 19 & 22 & $\mathrm{H}$ & France & Oui, Asie, Afrique & ND & non \\
\hline 20 & 74 & $\mathrm{H}$ & Maroc & oui & ND & non \\
\hline 21 & 63 & $\mathrm{~F}$ & France & Oui, Tunisie & non & non \\
\hline 22 & 46 & $\mathrm{H}$ & Maroc & oui & non & non \\
\hline$\overline{\mathrm{USa}}$ & 40,5 & $8 \mathrm{~F}(61 \%)$ & $\begin{array}{l}3(23 \%) \text { Maghreb } \\
1(7 \%) \text { Afrique }\end{array}$ & $5(38 \%)$ & ND & 0 \\
\hline 1 & 58 & $\mathrm{~F}$ & France & non & ND & non \\
\hline 2 & 28 & $\mathrm{~F}$ & France & Oui Thaïlande & ND & non \\
\hline 3 & 38 & $\mathrm{~F}$ & France & non & ND & ND \\
\hline 4 & 23 & $\mathrm{~F}$ & Maroc & oui & ND & non \\
\hline 5 & 39 & $\mathrm{M}$ & France & non & ND & non \\
\hline 6 & 53 & $\mathrm{~F}$ & Côte d'ivoire & oui & ND & non \\
\hline 7 & 25 & $\mathrm{~F}$ & France & non & ND & non \\
\hline 8 & 35 & $\mathrm{H}$ & Maroc & oui & ND & non \\
\hline 9 & 47 & $\mathrm{~F}$ & Turquie & non & ND & non \\
\hline 10 & 40 & $\mathrm{M}$ & Maroc & oui & ND & non \\
\hline 11 & 53 & $\mathrm{~F}$ & France & non & ND & ND \\
\hline 12 & 44 & $\mathrm{~F}$ & France & non & ND & non \\
\hline 13 & 43 & $\mathrm{H}$ & France & non & ND & ND \\
\hline USy & $59(44-79)$ & $9 \mathrm{H}(81 \%)$ & $\begin{array}{l}2(18 \%) \\
\text { Maghreb }\end{array}$ & $\begin{array}{l}3(28 \%) \\
\text { Afrique }\end{array}$ & ND & $2(18 \%)$ \\
\hline 1 & 55 & $\mathrm{~F}$ & Algérie & oui & ND & non \\
\hline 2 & 53 & $\mathrm{H}$ & France & non & ND & non \\
\hline 3 & 79 & $\mathrm{H}$ & France & oui, Tunisie & ND & non \\
\hline 4 & 44 & $\mathrm{H}$ & France & non & ND & oui \\
\hline 5 & 48 & $\mathrm{H}$ & France & non & ND & oui \\
\hline 6 & 44 & $\mathrm{H}$ & France & oui, Maroc & ND & non \\
\hline 7 & 54 & $\mathrm{H}$ & France & non & ND & non \\
\hline 8 & 45 & $\mathrm{H}$ & Cap vert & non & ND & non \\
\hline 9 & 57 & $\mathrm{~F}$ & France & non & ND & non \\
\hline 10 & 60 & $\mathrm{H}$ & France & non & ND & non \\
\hline 11 & 50 & $\mathrm{H}$ & Senegal & oui & ND & non \\
\hline
\end{tabular}

Tableau 1. Caractéristiques démographiques des patients

F : femme, H : homme, âge (en années), ND : donnée non disponible, ZE : zone d'endémie tuberculeuse, UT : uvéite tuberculeuse, Usa : uvéite sarcoïdosique, Usy : uvéite syphilitique 
2. Présentation clinique ophtalmologique:

Dans le groupe uvéite syphilitique, on comptait 4 (40\%) uvéites bilatérales, $6(60 \%)$ pan uvéites, 2 (20\%) uvéites postérieures et 2 antérieures. Les données étaient insuffisantes pour un patient. On trouvait $2(20 \%)$ uvéites récidivantes, 2 cataractes secondaires et $1(10 \%)$ uvéite hypertensive. Aucun nodule de Bouchut et aucune choroïdite serpigineuse n'était rapporté.

Dans le groupe uvéite sarcoïdosique, on trouvait 10 (77\%) uvéites bilatérales, plutôt intermédiaires (6 (46\%) d'entre-elles) versus $3(23 \%)$ antérieures et $5(38 \%)$ postérieures. 9 (69\%) uvéites étaient récidivantes, aucune hypertensive, mais $2(15 \%)$ avec une cataracte secondaire. Aucun nodule rapporté et aucune choroïdite serpigineuse.

Dans le groupe uvéite tuberculeuse, on trouvait 12 (55\%) uvéites bilatérales, 10 (45\%) postérieures, $12(55 \%)$ récidivante, $3(14 \%)$ hypertensive. Il y avait $8(36 \%)$ cataractes associées, $2(9,1 \%)$ choroïdites serpigineuses et 1 (4,5\%) nodule de Bouchut. 


\begin{tabular}{|c|c|c|c|c|c|c|c|c|c|c|c|c|}
\hline n & Uvéite & & & & & & & & & Choroïdite & Nodul & Cataracte \\
\hline & non & Oui & & & & & & & & & & \\
\hline & & unilat & bilat & ant & inter & post & pan & récidi & $\begin{array}{l}\text { HTA } \\
\end{array}$ & & & \\
\hline UT & 0 & $9 / 22$ & $13 / 22$ & $5 / 21$ & $1 / 20$ & $9 / 20$ & $6 / 20$ & $12 / 16$ & $3 / 14$ & $2 / 13$ & $1 / 13$ & $8 / 15$ \\
\hline 1 & - & - & + & + & - & - & - & + & + & - & - & + \\
\hline 2 & - & - & + & - & - & - & + & + & - & - & - & + \\
\hline 3 & - & - & + & - & - & - & + & + & - & + & - & - \\
\hline 4 & - & - & + & - & - & + & - & $\mathrm{ND}$ & ND & $\mathrm{ND}$ & $\mathrm{ND}$ & $\mathrm{ND}$ \\
\hline 5 & - & + & - & - & + & - & - & + & - & ND & $\mathrm{ND}$ & + \\
\hline 6 & - & + & - & - & - & - & + & + & - & - & - & + \\
\hline 7 & - & + & - & + & ND & ND & ND & + & + & $\mathrm{ND}$ & $\mathrm{ND}$ & $\mathrm{ND}$ \\
\hline 8 & - & - & + & - & - & + & - & $\mathrm{ND}$ & ND & ND & + & $\mathrm{ND}$ \\
\hline 9 & - & - & + & - & - & + & - & + & ND & ND & $\mathrm{ND}$ & $\mathrm{ND}$ \\
\hline 10 & - & - & + & - & - & - & + & + & ND & ND & $\mathrm{ND}$ & + \\
\hline 11 & - & + & - & - & - & + & - & - & - & - & - & - \\
\hline 12 & - & + & - & - & - & + & - & $\mathrm{ND}$ & ND & + & $\mathrm{ND}$ & $\mathrm{ND}$ \\
\hline 13 & - & - & + & + & - & - & - & + & + & - & - & + \\
\hline 14 & - & + & - & + & - & - & - & - & - & - & - & - \\
\hline 15 & - & - & + & - & - & - & + & ND & ND & ND & ND & ND \\
\hline 16 & & + & - & ND & ND & ND & ND & - & - & - & - & - \\
\hline 17 & - & - & + & + & - & - & - & $\mathrm{ND}$ & ND & ND & $\mathrm{ND}$ & $\mathrm{ND}$ \\
\hline 18 & - & - & + & - & - & - & + & $\mathrm{ND}$ & ND & ND & ND & + \\
\hline 19 & - & - & + & - & - & + & - & + & - & - & - & - \\
\hline 20 & - & + & - & - & - & + & - & + & - & - & - & + \\
\hline 21 & - & - & + & - & - & + & - & + & - & - & - & - \\
\hline 22 & - & + & - & - & - & + & - & - & - & - & - & - \\
\hline USa & 0 & $3 / 13$ & $10 / 13$ & $3 / 13$ & $6 / 13$ & $5 / 13$ & $2 / 13$ & $9 / 13$ & $0 / 13$ & $0 / 13$ & $0 / 13$ & $2 / 13$ \\
\hline 1 & - & - & + & + & - & - & - & + & - & - & - & - \\
\hline 2 & - & - & + & - & + & + & - & + & - & - & - & - \\
\hline 3 & - & - & + & - & - & - & + & + & - & - & - & + \\
\hline 4 & - & - & + & - & + & - & - & + & - & - & - & - \\
\hline 5 & - & - & + & - & + & - & - & + & - & - & - & - \\
\hline 6 & - & - & + & - & - & + & - & - & - & - & - & - \\
\hline 7 & - & + & - & - & - & + & - & - & - & - & - & - \\
\hline 8 & - & + & - & - & - & - & + & + & - & - & - & + \\
\hline 9 & - & - & + & + & - & + & - & + & - & - & - & - \\
\hline 10 & - & - & + & - & - & - & - & + & - & - & - & - \\
\hline 11 & - & + & - & + & + & - & - & - & - & - & - & - \\
\hline 12 & - & - & + & - & + & - & - & - & - & - & - & - \\
\hline 13 & - & - & + & - & + & + & - & + & - & - & - & - \\
\hline$\overline{\mathrm{USy}}$ & 0 & $3 / 10$ & $4 / 10$ & $2 / 10$ & $0 / 10$ & $2 / 10$ & $6 / 10$ & $2 / 10$ & $1 / 10$ & $0 / 10$ & $0 / 10$ & $2 / 10$ \\
\hline 1 & - & - & + & ND & ND & ND & ND & + & - & - & - & - \\
\hline 2 & - & - & - & - & - & - & + & - & - & - & - & - \\
\hline 3 & - & - & + & - & - & - & + & + & - & - & - & + \\
\hline 4 & - & - & + & + & - & + & - & - & - & - & - & - \\
\hline 5 & - & - & - & - & - & - & + & - & - & - & - & - \\
\hline 6 & - & - & + & - & - & - & + & - & - & - & - & - \\
\hline 7 & - & - & - & - & - & - & + & - & + & - & - & - \\
\hline 8 & - & + & - & - & - & - & + & - & - & - & - & + \\
\hline 9 & - & + & - & - & - & + & - & - & - & - & - & - \\
\hline 10 & ND & ND & ND & ND & ND & ND & ND & $\mathrm{ND}$ & ND & ND & $\mathrm{ND}$ & ND \\
\hline 11 & 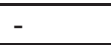 & + & - & + & - & - & - & - & - & - & - & - \\
\hline
\end{tabular}

Tableau 2. Présentation ophtalmologique

Uvéite unilat : unilatérale, bilat : bilatérale, ant : antérieure, int : intermédiaire, post : postérieure, pan : panuvéite, récidiv : récidivante, HTA : uvéite hypertensive, nodule : nodule de Bouchut, ND : donnée non disponible, UT : uvéite tuberculeuse, Usy : uvéite syphilitique, Usa : uvéite sarcoidosique, - : signe non présent, + : signe présent 


\begin{tabular}{|c|c|c|c|c|}
\hline $\mathrm{N}$ & Signes généraux & type & Signes pulmonaires & type \\
\hline UT & $9 / 22(41 \%)$ & & $3 / 22(14 \%)$ & \\
\hline 1 & - & - & - & - \\
\hline 2 & - & - & - & - \\
\hline 3 & + & $\begin{array}{l}\text { Adénopathie axillaire, } \\
\text { Fievre, arthrite }\end{array}$ & - & - \\
\hline 4 & - & - & - & - \\
\hline 5 & + & $-10 \mathrm{Kg}$ & - & - \\
\hline 6 & - & - & - & - \\
\hline 7 & + & Adénopathie axillaire & + & toux \\
\hline 8 & + & $-10 \mathrm{Kg}$ & - & - \\
\hline 9 & - & - & - & - \\
\hline 10 & - & - & - & - \\
\hline 11 & - & - & - & - \\
\hline 12 & + & $\begin{array}{l}\text { Adénopathie axillaire } \\
\text { Lésion cutanée nécrotique }\end{array}$ & - & - \\
\hline 13 & - & - & - & - \\
\hline 14 & + & Orchite récidivante & - & - \\
\hline 15 & + & Céphalées, hypoacousie & + & toux \\
\hline 16 & - & - & - & - \\
\hline 17 & + & $\begin{array}{l}-4 \mathrm{Kg} \\
\text { Erythème induré de Bazin }\end{array}$ & - & - \\
\hline 18 & - & - & + & pleurésie \\
\hline 19 & - & - & - & - \\
\hline 20 & - & - & - & - \\
\hline 21 & - & - & - & - \\
\hline 22 & + & $\begin{array}{l}\text { Sueurs, céphalées } \\
\text { HTA maligne }\end{array}$ & - & - \\
\hline USa & $7 / 13(54 \%)$ & - & - & 13 \\
\hline 1 & - & - & - & - \\
\hline 2 & + & Polyarthralgies, télangiectasies & - & - \\
\hline 3 & - & - & - & - \\
\hline 4 & + & $\begin{array}{l}\text { Paraplégie, vessie neurologique } \\
\text { Sarcoidose cérébrale et medullaire }\end{array}$ & - & - \\
\hline 5 & - & 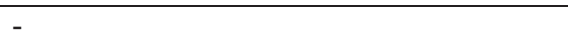 & - & - \\
\hline 6 & - & - & - & - \\
\hline 7 & + & Cicatrice chéloïde & - & - \\
\hline 8 & + & $\begin{array}{l}\text { Méningoencéphalite } \\
\text { rhombencéphalite }\end{array}$ & - & - \\
\hline 9 & + & érythème noueux & - & - \\
\hline 10 & + & Raynaud, arthralgies & - & - \\
\hline 11 & - & - & - & - \\
\hline 12 & - & - & - & - \\
\hline 13 & + & Arthralgies, adénopathie axillaire bilatérale & - & - \\
\hline USy & $7 / 11(63 \%)$ & - & 0 & 11 \\
\hline 1 & + & Eruption morbilliforme & - & - \\
\hline 2 & + & $\begin{array}{l}\text { Eruption morbilliforme } \\
\text { adénoapthies cervicales }\end{array}$ & - & - \\
\hline 3 & + & Méningite lymphocytaire & - & - \\
\hline 4 & + & $\begin{array}{l}\text { AEG, fièvre, } \\
\text { adénopathies inguinales, } \\
\text { Eruption morbilliforme } \\
\text { Leucoplasie chevelue de langue } \\
\text { Chancre }\end{array}$ & - & - \\
\hline 5 & - & - & - & - \\
\hline 6 & + & Eruption morbilliforme & - & - \\
\hline 7 & + & Eruption papulo-roséoliforme & - & - \\
\hline 8 & - & - & - & - \\
\hline 9 & + & $\begin{array}{l}\text { Arthralgies } \\
\text { Eruption morbilliforme }\end{array}$ & - & - \\
\hline 10 & - & - & - & - \\
\hline 11 & - & - & - & - \\
\hline
\end{tabular}

Tableau 3. Présentation systémique UT : uvéite tuberculeuse, Usy : uvéite syphilitique, Usa : uvéite sarcoïdosique 


\section{Présentation clinique systémique :}

$59 \%(13 / 22)$ des patients du groupe UT ne présentaient aucun signe systémique, alors que deux tiers des patients du groupe USy avaient une présentation clinique évocatrice de syphilis, tels qu'un chancre, une éruption cutanée morbilliforme, des adénopathies périphériques. 54\% (7/13) des patients du groupe Usa avaient une manifestation clinique de la sarcoïdose, tels qu'un érythème noueux ou des polyarthralgies.

\section{Résultats biologiques :}

On constatait dans le groupe UT que la majorité des patients avait un Quantiféron ${ }^{\circledR}$ positif $93 \%(15 / 16)$ ou une intra-dermo-réaction $86 \%(7 / 8)$ positif, contre respectivement $10 \%(1 / 10)$ et $50 \%(1 / 2)$ dans le groupe UNT. Les patients n'ayant pas bénéficié de Quantiféron ${ }^{\circledR}$ étaient globalement diagnostiqués avant 2006.

Le bilan hépatique montrait une cholestase chez 22,7\% (5/22) des patients UT et aucune dans les 2 autres groupes. $12,5 \%(2 / 16)$ des patients du groupe UT avaient une enzyme de conversion de l'angiotensine (ECA) positive contre 60\% (9/15) du groupe UNT (Tableau 4).

\section{L'imagerie :}

Dans le groupe UT, $81 \%(17 / 21)$ des patients avaient un scanner thoracique anormal, avec le plus souvent un ganglion, un nodule ou une excavation. Alors que le diagnostic de syphilis se faisant essentiellement sur la clinique, seuls 36\% (4/11) des patients avaient bénéficié d'un scanner dont la moitié étaient normaux. Le diagnostic différentiel était moins évident avec la sarcoïdose, puisque $60 \%(6 / 10)$ des patients avaient une adénopathie médiastinale au scanner mais aucun n'avait de nodule ou excavation. (Tableau 4) 


\section{La bactériologie et l'histologie}

6 patients du groupe UT et 6 patients du groupe Usa ont bénéficié d'une biopsie des glandes salivaires (BGSA). 5 patients du groupe UT ont eu une biopsie médiastinale, pulmonaire ou hépatique, ainsi que 6 patients du groupe Usa. 2 patients du groupe UT ont eu une ponction de chambre antérieure avec une PCR Mycobacterium tuberculosis. 9 patients du groupe USy ont eu une ponction lombaire ainsi que 2 patients du groupe UT.

\begin{tabular}{|c|c|c|c|c|}
\hline \multicolumn{2}{|l|}{ Groupe } & Tuberculose & Sarcoïdose & Syphilis \\
\hline \multicolumn{2}{|l|}{$\mathrm{n}$} & 22 & 13 & 11 \\
\hline \multicolumn{2}{|c|}{ Biologie standart } & 5 cholestases & 2cytolyses & 2cytolyses \\
\hline \multicolumn{2}{|c|}{ Hypergammaglobulinémie polyclonale } & 6 & 3 & 4 \\
\hline \multicolumn{2}{|l|}{ VIH } & 1 & 0 & 2 \\
\hline \multicolumn{2}{|l|}{ VHB } & 1 & 1 & 1 \\
\hline \multicolumn{2}{|l|}{ VHC } & 0 & 0 & 1 \\
\hline \multicolumn{2}{|l|}{ syphilis } & 0 & 0 & 11 \\
\hline \multicolumn{2}{|c|}{ toxoplasmose } & 9 & 1 & 3 \\
\hline \multicolumn{2}{|l|}{ ricketsie } & 0 & 0 & 0 \\
\hline \multicolumn{2}{|c|}{ toxocarose } & 2 & 0 & 2 \\
\hline \multicolumn{2}{|l|}{ ECA } & $2 / 16$ & $8 / 13$ & $1 / 4$ \\
\hline \multicolumn{2}{|l|}{ HLA } & $3 \mathrm{~B} 51$ & $1 \mathrm{~B} 51$ & 0 \\
\hline \multicolumn{2}{|c|}{ Quantiféron } & $15 / 16$ & $1 / 7$ & $0 / 3$ \\
\hline \multicolumn{2}{|c|}{ IDR tuberculine } & $7 / 8$ & 0 & $1 / 2$ \\
\hline \multirow[t]{5}{*}{ scanner } & normal & $4 / 21(19 \%)$ & $4 / 10(40 \%)$ & $2 / 4(50 \%)$ \\
\hline & ADP & $11 / 21(52,4 \%)$ & $6 / 10(60 \%)$ & $1 / 4(25 \%)$ \\
\hline & Syndrome interstitiel & $1 / 21(4,7 \%)$ & $2 / 10(20 \%)$ & $0 / 4$ \\
\hline & Nodule & $10 / 21(47,6 \%)$ & $0 / 10$ & $1 / 4(25 \%)$ \\
\hline & calcification & $1 / 21(4,7 \%)$ & $0 / 10$ & $0 / 4$ \\
\hline \multicolumn{2}{|l|}{ BGSA } & 5 sialadénite $/ 6$ & 5 sialadénite/ 6 & 0 \\
\hline \multicolumn{2}{|c|}{ Biopsie bronchique/ADPmédiastinale/hépatique } & $\begin{array}{l}\text { 2granulome avec } \\
\text { nécrose } / 5\end{array}$ & $\begin{array}{l}\text { 6granulome sans } \\
\text { nécrose/6 }\end{array}$ & 0 \\
\hline \multicolumn{2}{|c|}{ PCR sur PCA } & $1 / 2$ & 0 & 0 \\
\hline \multicolumn{2}{|l|}{ LBA } & $0 / 10$ & $0 / 1$ & $0 / 2$ \\
\hline \multicolumn{2}{|l|}{ LCR } & $1 / 2$ & 0 & $\begin{array}{l}\text { 3/9 méningite } \\
\text { lymphocytaire }\end{array}$ \\
\hline
\end{tabular}

Table 4. Présentation paraclinique

$\mathrm{n}$ : nombre, ECA : enzyme de conversion de l'angiotensine, HLA : typage HLA B51, IDR : intra-dermo-réaction, ADP : adénopathie médiastinale, BGSA : biopsie des glandes salivaires, biopsie bronchique/adénopathie médiastinale/hépatique : au moins une biopsie parmi celles-ci et mise en évidence ou non d'un granulome, PCR sur PCA : Polymérase Chain Reaction sur ponction de chambre antérieure, LBA : lavage bronchiolo-alvéolaire, LCR : liquide céphalo-rachidien par ponction lombaire 


\section{IV - Discussion}

Alors que le diagnostic de certitude des uvéites tuberculeuses reste impossible dans la majorité des cas, il n'existe toujours pas de consensus sur l'attitude diagnostique et thérapeutique à adopter dans ce domaine. Comme le montre Lou SM. et al. [16, 17], les pratiques diffèrent en fonction des médecins et selon les régions du Monde. Face à un patient avec une uvéite granulomateuse, la conduite des praticiens est très aléatoire et varie aussi au sein du même pays (dans cette étude, les médecins du Nord des EtatsUnis n'avaient pas les mêmes habitudes de prescriptions que ceux du Sud). Ceci a pour conséquence directe, une discordance dans la prise en charge étiologique et donc thérapeutique des patients atteints d'uvéite. Il paraît dès lors indispensable de proposer des critères diagnostics d'uvéite tuberculeuse, conduisant à la prescription d'un traitement antituberculeux d'épreuve, afin d'uniformiser notre pratique et de soigner le maximum de patients.

Dans notre étude, il ressort que l'âge, l'origine ou le vécu dans une zone d'endémie tuberculeuse, la négativité de l'enzyme de conversion de l'angiotensine, le test IGRA positif et la présence d'un nodule ou d'une excavation au scanner thoracique sont des critères statistiquement significatifs d'uvéite tuberculeuse. (Tableau 5).

Nos patients avaient en effet un âge moyen de 57,9 ans, soit plus âgés que ceux non atteints d'uvéite tuberculeuse ( $\mathrm{p}=0,0079)$, mais en rapport probablement avec l'âge jeune des uvéites sarcoïdosiques. Cette donnée est contradictoire par rapport à la série anglaise de Manousaridis K. et al. [18] à Newcastle où l'âge moyen est de 46 ans, ou celle de Sanghi C. et al. [21] à Manchester, de 36 ans. Ceci peut être expliqué par le fait que nos patients sont en moyenne plus âgés sur la Côte d'Azur que dans le reste du Monde et que par conséquent c'est la différence d'âge au sein de notre population qui est importante et rend ce critère diagnostic valide. Mais les 2 études anglaises n'avaient, elles, pas de population témoin. 
Le sexe n'est pas apparu comme critère diagnostic, en accord avec les données contradictoires des 2 séries anglaises, qui comptaient respectivement $71 \%$ et $41 \%$ d'hommes [18, 21].

L'origine ou le vécu dans une zone d'endémie tuberculeuse est un critère significatif d'uvéite tuberculeuse : $90 \%$ de nos patients tuberculeux étaient concernés, alors que cela implique seulement $38 \%$ des patients avec sarcoïdose et $28 \%$ des patients syphilitiques $(\mathrm{p}<0,0001)$. Ceci vient conforter les données de la littérature [6]. On peut toutefois relever que nos patients étaient majoritairement originaires d'Afrique du Nord (36\%) et d'Afrique Noire (23\%), alors que dans les 2 séries anglaises, on retrouvait respectivement $52 \%$ d'Asiatiques et $10 \%$ d'Africains à Newcastle et $55 \%$ d'Asiatiques et $18 \%$ d'Africains à Manchester. Ces données sont bien sûr en rapport avec une immigration majoritairement indienne et pakistanaise en Angleterre, maghrébine et africaine en France.

Il est aussi important de souligner que malgré un patient séropositif pour le VIH, et trois patients ayant reçu préalablement un immunosuppresseur (ciclosporine pour une maladie de Behçet diagnostiquée par défaut et une Sclérose en plaque, étanercept pour une spondylarthrite ankylosante), les patients avec une uvéite tuberculeuse ne sont pas plus immunodéprimés que les autres, tout comme dans la cohorte de Newcastle [18]. Bien qu'on décrive $59 \%$ d'uvéites bilatérales et $45 \%$ d'uvéites postérieures dans notre groupe d'uvéites tuberculeuses, cette présentation clinique n'est pas spécifique, cela corrobore les données controversées de la littérature: la présentation clinique ophtalmologique diffère en fonction des études. Même si Gupta A. et al. [12] en 2010, décrit que la présence de synéchies postérieures, de vascularite rétinienne ou de choroïdite serpigineuse sont en faveur d'une uvéite tuberculeuse, ces éléments sont rarement présents dans la description clinique et laissent encore échapper trop de diagnostics. Le caractère récidivant de l'uvéite et l'association à une cataracte sont les seuls éléments de la présentation ophtalmologique qui attirent notre attention et orientent vers une uvéite tuberculeuse, sans pour autant être statistiquement 
significatifs ( $\mathrm{p}=0,0897$ et $\mathrm{p}=0,0326$ respectivement). Ceci peut être expliqué par le mécanisme réactionnel de l'uvéite et la présence probable d'une inflammation chronique, favorisant l'apparition d'une cataracte. L'âge plus élevé des patients avec UT peut toutefois être un biais et expliquer la présence plus importante de cataracte dans ce groupe. De plus nos patients tuberculeux ne présentaient pas plus de signes cliniques généraux ou pulmonaires que les autres, sans doute lié au fait que l'uvéite tuberculeuse rentre principalement dans le cadre d'une tuberculose latente. Dans la cohorte de Newcastle, seulement 2 patients avec une tuberculose active présentaient des signes généraux [18].

Sur le plan biologique, la non élévation de l'enzyme de conversion de l'angiotensine (ECA) est un critère diagnostic significatif. Pourtant l'ECA est produite par les macrophages et les cellules épithélioïdes présents dans les granulomes et peut donc être un marqueur de sarcoïdose mais aussi de tuberculose selon certains auteurs. Plutôt en accord avec l'étude indienne de Sainani G. et al. [20] et l'étude chinoise de Zangh Y. et al. [24], nous montrons que l'ECA est même plus élevée dans les cas de sarcoïdose. Dans le groupe UT, 2 patients avaient une ECA positive, l'un présentait une adénite granulomateuse et l'autre une méningite lymphocytaire mais pas de granulome. Et parmi les 8 patients du groupe Usa avec une ECA positive, 6 avaient un granulome. Un patient du groupe USy avait une ECA positive mais n'avait pas bénéficié d'histologie. Nous relevons également, sans pouvoir l'expliquer, la présence plus importante d'une cholestase chez les patients du groupe UT $(22,7 \%$ versus $0 \%$, $\mathrm{p}=0,0036)$.

Le test IGRA (Quantiféron ${ }^{\circ}$ ) est bien sûr un critère diagnostic majeur de tuberculose, puisque déjà évalué à plusieurs reprises dans la littérature $[15,19,22]$. Dans notre cohorte, le seul patient atteint d'uvéite tuberculeuse avec un Quantiféron ${ }^{\circ}$ négatif, n'avait pas eu d'intradermo-réaction, mais présentait un nodule pulmonaire au scanner. Ce patient était porteur d'un syndrome myélodysplasique, entrainant une lymphopénie chronique, qui rend le test IGRA ininterprétable dans le contexte. Une 
patiente du groupe UNT avait un Quantiféron positif. Cette patiente avait initialement été traitée par antituberculeux. Mais devant l'absence de réponse clinique, elle a été considérée comme "sarcoïdose », a reçu des corticoïdes puis a guéri après la mise sous immunosuppresseur (cyclophosphamide puis méthotrexate). Concernant l'intradermo-réaction à la tuberculine, l'analyse statistique n'a pu être réalisée en raison du manque de données. (En effet les patients ayant bénéficié d'une intradermoréaction seule étaient diagnostiqués avant 2010 et uniquement 2 patients sans uvéite tuberculeuse avaient été testés). Mais comme le montre Ball PM. et al. [3] en 2010, le Quantiféron $^{\circ}$ a une valeur prédictive positive supérieure à l'intradermo-réaction dans le diagnostic des uvéites chroniques. Il est donc licite d'analyser le test IGRA seul. Un test Quantiféron TB Gold (méthode en tube, Cellestis) mesure par méthode ELISA, la quantité d'INF-g sécrétée par les lymphocytes T récoltés dans $1 \mathrm{ml}$ de sang total, après stimulation in vitro par les antigènes ESAT-6, CFP-10 et TB7.7 (peptides synthétiques représentant des protéines du Mycobactérium tuberculosis). Il s'agit donc d'un test peu sensible mais spécifique, négatif même en cas de BCG (car les peptides ne sont pas présents dans le vaccin, ni dans les autres mycobactéries sauf M. kansasii, M. szulgai et $M$. marinum, mais non pathogènes sauf en cas de VIH ou d'immunodépression sévère) [6]. Il ne permet donc pas de dater l'infection et ne fait pas la différence entre une tuberculose active ou latente. Sa réalisation est donc recommandée uniquement en cas d'histoire évocatrice de tuberculose. On ne peut donc réduire le diagnostic d'uvéite tuberculeuse à ce seul test.

Enfin comme le montrait Ganesh SK. et al. [8] en 2011, le scanner thoracique est utile à l'orientation diagnostique des uvéites tuberculeuses. Alors qu'il était anormal dans $81 \%$ de leurs cas avec des aspects évocateurs de tuberculose, notre étude le confirme avec le même pourcentage d'anomalies (17 patients sur 22). C'est la présence d'un nodule pulmonaire $(47,6 \%$ versus $6,67 \%, p=0,0112)$ qui est le plus spécifique de tuberculose, puisque les adénopathies médiastinales sont aussi présentes dans la sarcoïdose. Enfin la PCR BK sur ponction de chambre antérieure réalisée dans moins 
de $10 \%$ des cas ne peut pour l'instant pas faire partie des critères diagnostics d'uvéites tuberculeuses, en raison de son manque de sensibilité.

De par sa rétrospectivité, notre étude est bien sûr critiquable et comporte plusieurs biais. Les antécédents familiaux, la notion de contage et l'antériorité de vaccination par le BCG n'ont pu être recensés en raison du biais de mémoire. A Newcastle, 24\% de contage a été rapporté, alors que Campos WR. et al. [7] en 2008 montre qu'un antécédent de contage est facteur de risque d'uvéite tuberculeuse (OR 10,67 mais avec un intervalle de confiance comportant 1). Certains critères, comme l'uvéite récidivante et la présence d'une cataracte associée, qui auraient pu apparaître comme significatifs n'ont pu être validés par manque de puissance. Notre série est certes peu importante, mais c'est le cas de presque toutes les études sur le sujet, en dehors des études de cohorte diagnostique par intradermo-réaction et test IGRA, [1, 2, 3, 9, 14, 19], les uvéites tuberculeuses restant une pathologie rare. De plus, tous les patients n'ont pas bénéficié du même bilan diagnostic, puisqu'il s'agit d'une étude rétrospective. Nous n'avons donc pas pu analyser les données en analyse multi variée et en tirer un score diagnostic statistique.

Cependant l'instinct clinique et la significativité des résultats en analyse uni variée suffisent à établir des critères diagnostics malgré le peu de patients que nous avions. Enfin, seule la réponse au traitement antituberculeux valide nos résultats. Un recul suffisant était donc nécessaire pour constater la guérison. Les patients recevant au moins six mois d'antibiothérapie, nous avons arrêté les inclusions en juillet 2014. Il n'existe actuellement pas de consensus concernant le traitement des uvéites tuberculeuses. Il est habituel de réaliser une quadri-antibiothérapie antituberculeuse pendant 2 mois (plus si forme résistante) puis 4 à 7 mois de bithérapie sauf si une atteinte neurologique est présente, le traitement est alors plus long. Ici, 4 patients ont reçu une quadri-antibiothérapie et 18 une tri-antibiothérapie antituberculeuse, associée pour 7 patients à une corticothérapie systémique et pour 11 d'entre-eux à une corticothérapie locale. La réponse au traitement était évaluée à 2 mois, puis un relais 
par isoniazide et rifampicine était réalisé, maintenu pendant 4 à 10 mois selon l'efficacité. 16 patients avaient une amélioration ou une stabilisation de l'uvéite à 2 mois et tous étaient guéris à la fin de l'étude.

Enfin il est important de souligner que notre étude est une des premières séries françaises descriptives d'uvéite tuberculeuse et la seule de Provence-Alpes-Côte d'Azur. Cette région se caractérise par l'une des plus fortes incidences tuberculeuses, ce qui peut rendre l'extrapolation de nos résultats à d'autres populations plus difficile. L'Institut National de Veille Sanitaire rapporte en effet une survenue maximale de 16,3 pour 100000 habitants de tuberculose en Ile-de-France et de 8,0 pour 100000 habitants en PACA en 2010 (BEH 24-25, 12 juin 2012). On peut citer la cohorte prospective de Lino A. et al. [15] qui réalise en 2010 à l'hôpital Saint-Antoine à Paris un bilan exhaustif à 25 patients avec une uvéite sans étiologie évidente, et montre l'intérêt du test IGRA, pour éliminer les uvéites tuberculeuses. Notre travail va plus loin, puisqu'il compare les uvéites tuberculeuses aux uvéites non tuberculeuses, sur le terrain, la présentation clinique et paraclinique. Bien que notre étude soit rétrospective et donc limitée, c'est la seule qui combine tous les éléments de diagnostic et montre une différence par rapport à une population témoin. Bien que Campos WR. et al. [7] avait déjà tenté ce travail, au Brésil, il ne montrait une différence que sur l'antécédent de contage. Nous confirmons donc l'impression antérieure que l'origine ou le vécu en zone d'endémie tuberculeuse, le test IGRA positif et la présence d'un nodule pulmonaire au scanner sont des paramètres en faveur d'une uvéite tuberculeuse. Nous apprenons aussi que l'âge plus élevé, la négativité de l'ECA et la présence éventuelle d'une cholestase au bilan biologique, orientent vers une uvéite tuberculeuse. L'absence de signe clinique ophtalmologique évocateur plaide pour une consultation de médecine interne. Nous recommandons dès lors en l'absence d'un tableau clinique évident, un bilan étiologique exhaustif, qui cherchera une origine ou un vécu en zone d'endémie tuberculeuse, la réalisation de sérologies virales et d'une ECA en première intention. En l'absence de cause évidente (herpès, toxoplasmose, syphilis), le scanner 
thoracique (car plus sensible que la radiographie [14]) et le test IGRA doivent être réalisés, ce qui permet un screening plus adapté et conduit à un plus grand nombre de diagnostics d'uvéite tuberculeuse.

\section{$\mathrm{V}$ - Conclusion}

Notre étude permet dès lors de dégager des critères diagnostics très fortement évocateurs d'uvéite tuberculeuse. Ainsi face à un patient avec une uvéite granulomateuse ou compatible de plus de 50 ans, ayant vécu en pays d'endémie tuberculeuse, avec un test IGRA positif et un nodule pulmonaire au scanner, une enzyme de conversion négative, on peut conclure à une uvéite tuberculeuse jusqu'à preuve du contraire et initier un traitement antituberculeux. 


\begin{tabular}{|c|c|c|c|c|}
\hline & Variable & UT & UNT & $\begin{array}{l}\mathrm{p} \text {-value (test } \\
\text { univarié) }\end{array}$ \\
\hline \multirow{5}{*}{ Epidémiologie } & Age (moyenne) & 57,9 & 46,46 & 0,0079 \\
\hline & Hommes & $55,5 \%$ & $54,17 \%$ & \multirow[t]{2}{*}{0,9794} \\
\hline & Femmes & $45,45 \%$ & $45,83 \%$ & \\
\hline & Origine pays endémie & $71 \%$ & $25 \%$ & 0,0002 \\
\hline & Voyage pays endémie & $90 \%$ & $47,6 \%$ & $<0,0001$ \\
\hline \multirow{11}{*}{$\begin{array}{l}\text { Présentation clinique } \\
\text { ophtalmologique }\end{array}$} & Uvéite unilatérale & $40,9 \%$ & $26,09 \%$ & 0,2917 \\
\hline & Uvéite bilatérale & $59,09 \%$ & $60,87 \%$ & 0,9031 \\
\hline & Antérieure & $23,81 \%$ & $22,73 \%$ & 1 \\
\hline & Intermédiaire & $5 \%$ & $27,27 \%$ & 0,0961 \\
\hline & Postérieure & $45 \%$ & $31,82 \%$ & 0,3796 \\
\hline & Panuvéite & $30 \%$ & $36,36 \%$ & 0,6622 \\
\hline & Récidivante & $75 \%$ & $47,83 \%$ & 0,0897 \\
\hline & Hypertensive & $21,43 \%$ & $4,35 \%$ & 0,1419 \\
\hline & Choroidite serpigineuse & $15,38 \%$ & 0 & 0,1238 \\
\hline & Nodule de Bouchut & $7,69 \%$ & 0 & 0,3611 \\
\hline & Cataracte associée & $53,33 \%$ & $17,39 \%$ & 0,0326 \\
\hline \multirow{2}{*}{$\begin{array}{l}\text { Présentation clinique } \\
\text { systémique }\end{array}$} & Signes généraux & $40,91 \%$ & $58,33 \%$ & 0,2377 \\
\hline & Signes pulmonaires & $13,64 \%$ & 0 & 0,1014 \\
\hline \multirow{6}{*}{ Biologie } & Cholestase & $22,7 \%$ & $\mathbf{0}$ & 0,0036 \\
\hline & Hypergammaglobulinémie & $27,27 \%$ & $36,84 \%$ & 0,5114 \\
\hline & VIH & $4,76 \%$ & $9,52 \%$ & 1 \\
\hline & VHB & $4,65 \%$ & $8,70 \%$ & 1 \\
\hline & VHC & 0 & $4,35 \%$ & 1 \\
\hline & ECA élevée & $12,5 \%$ & $60 \%$ & 0,0091 \\
\hline \multirow{2}{*}{ Tests diagnostics } & Quantiféron & $93,75 \%$ & $10 \%$ & $7,097.10^{\wedge}-5$ \\
\hline & IDR & $87,5 \%$ & $50 \%$ & NR \\
\hline \multirow{4}{*}{ Imagerie } & Scanner normal & $19,05 \%$ & $40 \%$ & 0,26 \\
\hline & Ganglion & $52,38 \%$ & $46,67 \%$ & 1 \\
\hline & Nodule/excavation & $47,6 \%$ & $6,67 \%$ & 0,0112 \\
\hline & Syndrome interstitiel & $4,76 \%$ & $13,33 \%$ & 0,5588 \\
\hline
\end{tabular}

Tableau 5. Critères diagnostics des uvéites tuberculeuses

Age (en années), UT : uvéite tuberculeuse, UNT : uvéite non tuberculeuse, ECA : enzyme de conversion de l'angiotensine, IDR : intra-dermo-réaction à la tuberculine 


\section{VI - Critères diagnostics des uvéites tuberculeuses}

\section{Résumé :}

Introduction : La tuberculose est un problème de santé publique mondiale mais qui, lorsqu'elle est diagnostiquée peut être traitée efficacement. $\%$ d'entre-elles peuvent se révéler par une uvéite, dont le diagnostic de certitude pose encore de nombreuses difficultés. Malgré de nombreuses études sur le sujet, aucune ne cumule l'évaluation de tous les moyens diagnostics disponibles. Nous avons souhaité établir des critères diagnostics d'uvéite tuberculeuse permettant d'orienter la pratique clinique, à partir d'une étude descriptive comparant les uvéites tuberculeuses (UT) à d'autres uvéites granulomateuses non tuberculeuses (UNT) (sarcoïdose et syphilis uniquement).

Méthodes : 22 patients avec une uvéite tuberculeuse, 11 avec une uvéite syphilitique et 13 avec une uvéite sarcoïdosique, suivis conjointement entre l'ophtalmologie et la médecine interne d'un centre labellisé ont été inclus entre 2004 et 2014. Pour chaque patient, des données épidémiologiques, la présentation clinique ophtalmologique et systémique, le profil biologique, le résultat de l'intradermoréaction, de l'INFg Release Assay (IGRA), les examens d'imagerie et la réalisation éventuelle d'une histologie ont été recueillis de façon rétrospective. Chaque variable a ensuite été comparée entre les 2 groupes (UT versus UNT) afin de déterminer des critères diagnostics d'uvéite tuberculeuse.

Résultats : Les UT avaient un âge moyen de 57,9 ans contre 46,5 ans dans le groupe UNT ( $\mathrm{p}=0,0079$ ). 19 patients sur $22(90 \%)$ étaient d'origine ou avaient vécu dans un pays d'endémie tuberculeuse pour les UT contre $3 / 11$ (28\%) des uvéites syphilitiques et 5/13 (38\%) des uvéites sarcoïdosiques ( $\mathrm{p}=0,0002)$. Il n'y avait pas de différence significative sur la localisation de l'uvéite (uni ou bilatérale, antérieure, intermédiaire ou postérieure). On constatait toutefois plus d'uvéites récidivantes 12/22 (55\%) $(\mathrm{p}=0,0897)$ et de cataractes associées $8 / 22(36 \%)(\mathrm{p}=0,0326)$ dans le groupe UT mais de façon non significative. 15/16 (93\%) patients du groupe UT avaient un test IGRA positif contre 0/3 dans le groupe syphilis et $1 / 7$ dans le groupe sarcoïdose $(\mathrm{p}<0,0001) .10 / 21(48 \%)$ des UT avaient un nodule au scanner thoracique contre $1 / 4$ des syphilis et $0 / 10$ des sarcoïdoses $(p=0,0112)$. A l'inverse, $2 / 16(12,5 \%)$ patients des UT avaient une enzyme de conversion de l'angiotensine positive, contre 8/13 (61\%) des sarcoïdoses et $1 / 11(9 \%)$ des syphilis $(\mathrm{p}=0,0091)$.

Discussion : Dans notre étude, la présentation clinique ophtalmologique des uvéites tuberculeuses n'est pas spécifique, à l'instar de la littérature. Ceci plaide donc en faveur de la nécessité d'un bilan complémentaire plus exhaustif. Alors même que la dernière revue américaine de Susan M. Lou et al. parue en juillet 2014 montre qu'il n'existe toujours pas de consensus concernant l'attitude diagnostique des praticiens devant une uvéite granulomatose, nous démontrons qu'un screening adapté d'un certain type de population permet d'arriver plus souvent au diagnostic de tuberculose latente à manifestation ophtalmologique. Enfin, l'évolution à chaque fois favorable sous traitement anti- tuberculeux seul confirme notre travail.

Conclusion: Notre étude permet par contre de dégager des critères diagnostics très fortement évocateurs d'uvéite tuberculeuse. Ainsi face à un patient avec une uvéite granulomateuse de plus de 50 ans, ayant vécu en pays d'endémie tuberculeuse, avec un test IGRA positif et un nodule pulmonaire au scanner, une enzyme de conversion négative, on peut conclure à une uvéite tuberculeuse jusqu'à preuve du contraire et initier un traitement anti-tuberculeux.

Mots-clés : uvéite, tuberculose, quantiféron, granulomatose 


\section{VII - Références Bibliographiques}

1. ANG, Marcus. Diagnosis of tuberculous uveitis: clinical application of an interferon-gamma release assay. Ophthalmology. juillet 2009, vol.116, nº , p.1391-1396.

2. ANG, Marcus. Interferon-gamma release assay as a diagnostic test for tuberculosis-associated uveitis. Eye (Lond). mai 2012, vol.26, n5, p.658-665.

3. BALL, Pierre-Marie. Usefulness of an in-vitro tuberculosis interferon- \& release assay (T-SPOT.TB) in the first-line check-up of uveitis patients. Annals of Medicine. octobre 2010, vol.42, nº 7, p.546-554.

4. BODAGHI, Bahram. Uvéite. Atlas en ophtalmologie. Elsevier Eds. 2009. 394p.

5. BOURCIER, Tristan. Les infections oculaires. Rapport annuel des sociétés d'ophtalmologie de France : Bsof. 2010. 432p.

6. BREZIN, Antoine. Les uvéites. Société française d'ophtalmologie. Masson. 2010. 760p.

7. CAMPOS, Wesley Ribeiro. Tuberculous uveitis at a referral center in southeastern Brazil. Journal Brasilian of Pneumology. février 2008, vol.34, n², p.98-102.

8. GANESH, Sudha K. Role of high-resolution computerized tomography (HRCT) of the chest in granulomatous uveitis: a tertiary uveitis clinic experience from India. Ocular Immunology Inflammation. février 2011, vol.19, $\mathrm{n}^{\circ} 1$, p.51-57.

9. GINEYS, Raquel. QuantiFERON-TB gold cut-off value: implications for the management of tuberculosis-related ocular inflammation. American Journal of Ophthalmology. Septembre 2011, vol.152, n³, p.433-440.

10. GRAHAM, SM. Evaluation of tuberculosis diagnostics in children, 1: proposed clinical case definitions for classification of intrathoracic tubercu- losis disease. Consensus from an Expert Panel. Journal of Infectious Disease. 2012 , vol. 205, p.199-208.

11. GUPTA, Avisek. Classification of intraocular Tuberculosis. Ocular Immunology Inflammation. 2015 , vol.23, $\mathrm{N}^{\circ} 1$, p.7-13.

12. GUPTA, Avisek. Ocular signs predictive of tubercular uveitis. American Journal of Ophthalmology. avril 2010, vol.149, n4, p.562-570.

13. GUPTA, Vassali. Clinics of ocular Tuberculosis. Ocular Immunololy Inflammation. 2015, vol.23, $\mathrm{n}^{\circ} 1, \mathrm{p} .14-24$.

14. HONG, Bryan Kun. The utility of routine tuberculosis screening in county hospital patients with uveitis. British Journal of Ophthalmology. août 2014, vol.98, n8, p.1091-5.

15. LINO, Anne. Diagnostic des uvéites tuberculeuses: intérêt des tests interférons gamma. Revue des Maladies Respiratoires. mars 2011, vol.28, n³, p.317-321.

16. LOU, Susan. members of the Uveitis Specialists Study Group. Diagnosis and Treatment for Ocular Tuberculosis among Uveitis Specialists: The International Perspective. Ocular Immunology Inflammation. 2015, vol.23, $\mathrm{n}^{\circ} 1, \mathrm{p} .32-39$

17. LOU, Susan M. Lack of Consensus in the Diagnosis and Treatment for Ocular Tuberculosis among Uveitis Specialists. Ocular Immunology Inflammation. juillet 2014, p.1-7. 
18. MANOUSARIDIS, Kleanthis. Clinical presentation, treatment, and outcomes in presumed intraocular tuberculosis: experience from Newcastle upon Tyne, UK. Eye (Lond). Avril 2013, vol.27, n4, p.480-486.

19. MODORATI, Giulio. Clinical application of an in-house ELISPOT assay in patients with suspicious tuberculous uveitis and no signs of active tuberculosis. European Journal of Ophthalmology. octobre 2012, vol.22, n5, p.808-813.

20. SAINANI, GS. Serum angiotensin converting enzyme activity in sarcoidosis and pulmonary tuberculosis. Journal Association Physicians India. Janvier 1996, vol.44, n¹, p.29-30.

21. SANGHVI, Kamlesh. Presumed tuberculous uveitis: diagnosis, management, and outcome. Eye (Lond). avril 2011, vol.25, $\mathrm{n}^{\circ} 4$, p.475-480.

22. SUDHARSHAN, Sekar. Utility of QuantiFERON®-TB Gold test in diagnosis and management of suspected tubercular uveitis in India. International Ophthalmology. juin 2012, vol. 32, n³, p.217-223.

23. VASCONCELOS-SANTOS Daniel V. Strengths and Weaknesses of Diagnostic Tools for Tuberculous. Ocular Immunology Inflammation. 2009, vol.17, n5, p.351-355.

24. ZHANG, Yonggang. Association between ACE I/D polymorphism and pulmonary tuberculosis in Chinese population. Molecular Biology Reports. Mai 2014, vol.41, n5, p.3187. 


\section{VIII - Annexes}

\section{Annexe 1}

\section{Bibliographie complémentaire}

25. ANG, Marcus. Discordance of two gamma-release assays and tuberculin skin test in patients with uveitis. British Journal of Ophtalmology. Juillet 2014, vol.98, p.1649-1653.

BABU, Kalpana. Predictors for tubercular uveitis: a comparison between biopsy-proven cases of tubercular and sarcoid uveitis. Retina (Philadelphia, Pa). mai 2012, vol.32, n5, p.1017-1020.

BASU, Soumyava. Tuberculous uveitis. Ophthalmology. mai 2010, vol.117, n 5, p.1049-1050.

CIMINO, Luca. Tuberculous uveitis, a resurgent and underdiagnosed disease. Internal Ophthalmology. avril 2009, vol.29, n², p.67-74.

KIM, Sung-Han. Diagnostic usefulness of a T-cell-based assay for extrapulmonary tuberculosis in immunocompromised patients. American Journal of Medicine. février 2009, vol.122, n², p.189-195.

KOMIYA, Kosaku. Impact of peripheral lymphocyte count on the sensitivity of 2 IFN-gamma release assays, QFT-G and ELISPOT, in patients with pulmonary tuberculosis. Internal Medicine. 2010, vol.49, n 17 , p.1849-1855.

31. LA DISTIA, Nora. Clinical manifestations of patients with intraocular inflammation and positive QuantiFERON-TB gold in-tube test in a country nonendemic for tuberculosis. American Journal of Ophthalmology. avril 2014, vol.157, nº4, p.754-761.

32. PATEL, Sarju S. Mycobacterial ocular inflammation: delay in diagnosis and other factors impacting morbidity. JAMA Ophthalmology. juin 2013, vol.131, nº, p.752-758.

33. PONCE DE LEON, Dorio. Comparison of an interferon-gamma assay with tuberculin skin testing for detection of tuberculosis (TB) infection in patients with rheumatoid arthritis in a TB-endemic population. Journal of Rheumatology. mai 2008, vol.35, n5, p.776-781.

34. SHARMA, Kusum. Loop-mediated isothermal amplification for rapid diagnosis of tubercular uveitis. JAMA Ophthalmology. juin 2014, vol.132, nº, p.777-778.

35. VOS, AG. Diagnosis and treatment of tuberculous uveitis in a low endemic setting. Internal Journal Infectious Disease. novembre 2013, vol.17, n¹1, p.993-999. 


\section{Annexe 2}

Article en cours de soumission pour Annals of Internal Medicine

Title: Diagnostic Criteria Of Tuberculous Uveitis: a clinical comparative study of granulomatous uveitis

Running Title: Diagnostic Criteria Of Tuberculous Uveitis

Sarah Lechtman ${ }^{1}$, Viviane Queyrel ${ }^{1}$, Célia Maschi ${ }^{2}$, Jean-Gabriel Fuzibet ${ }^{1}$, Pierre Gastaud ${ }^{2}$, Stéphanie Baillif $^{2}$, Nathalie Tieulié ${ }^{1}$

1: Department of internal Medicine, L'Archet 1 Hospital, Nice, France

2: Department of ophthalmology, Saint Roch Hospital, Nice, France

Word count: 3498

Tables: 5

Grants and financial support: None

\section{Corresponding Author}

Dr Nathalie Tieulié, MD

Hôpital L'archet 1, 151 route Saint Antoine Ginestière, 06202 Nice

Tel : 0492035412

Mail: tieulie.n@chu-nice.fr 


\section{Diagnostic Criteria Of Tuberculous Uveitis:}

\section{A clinical comparative study of granulomatous uveitis}

\section{Abstract:}

Background: No consensus exists regarding the clinical signs and tests confirming the diagnosis of intraocular tuberculosis.

Objective: To determine diagnostic criteria for tuberculous uveitis by comparing successfully treated tuberculous uveitis (TBU) to other cases of non-tuberculous granulomatous uveitis (UNT)

Design: Retrospective study

Setting: Nice University Hospital, Nice, France

Patients: Twenty-two tuberculous uveitis, 11 syphilitic, and 13 sarcoid uveitis cases were analysed. For each patient, epidemiological, clinical and biological criteria were reviewed. Results of the Mantoux reaction, interferon-gamma release assays (IGRA) and CT chest scan were collected.

Results: Ninety per cent of TBU patients lived in a TB-endemic region versus $47 \%$ of UNT patients (p $=0.0002)$. No significant difference in the clinical presentation of uveitis was found between groups. 15/16 TBU patients had a positive IGRA versus $1 / 10$ in the UNT group $(\mathrm{p}<0,001) .10 / 21(47 \%)$ of the TBU patients had lung nodules on chest CT scan versus $1 / 14(6.7 \%)$ of UNT patients $(\mathrm{p}=0.0112)$.

Limitations: Ocular signs of tuberculous uveitis are not specific. Complementary tests are needed to confirm the diagnosis.

Conclusion: In the absence of diagnostic gold standard test, the TB-endemic region origin, a positive IGRA test and lung lesions on chest CT scan in a patient with granulomatous uveitis should be diagnosed with tuberculous uveitis and a therapeutic test with antitubercular therapy should be initiated.

Registration: None

Funding Source: None 


\section{Introduction}

Mycobacterium tuberculosis infects one-third of the world population, but causes a disease in $10 \%$ of the infected individuals. Tuberculosis remains a public health problem and has been declared by the World Health Organization as "planetary emergency medical condition" in 1993, mainly affecting Asia and sub-Saharan Africa [1]. If properly diagnosed, tuberculosis can be successfully treated.

In $90 \%$ of cases, the bacillus tubercle remains quiescent without any symptoms: it is latent tuberculosis. The occurrence of uveitis in such a context should be interpreted with caution. Ocular tuberculosis represents only $2 \%$ of uveitis cases, while $50 \%$ are related to idiopathic uveitis [2]. However, $18 \%$ of tuberculosis patients disclose ocular signs, if an ophthalmic examination is performed systematically. Ocular lesions in tuberculosis would result from two different mechanisms: either from an intraocular infection by mycobacteria (haematogenous spread of a tuberculosis disease), or as a type IV hypersensitivity reaction (reactivation during an extra-ocular latent tuberculosis) [3].

The clinical signs of intraocular tuberculosis have wide variation in anatomic location and morphological appearance. All ocular structures may be affected [4]. Some clinical signs are consistent with intraocular tuberculosis such as: anterior or posterior uveitis willingly granulomatous and bilateral, granulomatous or serpiginous choroiditis, choroidal tubercles. An optic neuritis or retinal vasculitis may also be associated. But tuberculosis may also affect external ocular tissues (eyelids, lacrimal gland, conjunctiva) masquerading several infective or non-infective diseases.

The diagnosis of tuberculous uveitis is challenging. Rarely, the presence of Mycobacterium tuberculosis DNA on aqueous humor samples confirms the diagnosis [5]. Most of the patients have no history of systemic tuberculosis and their clinical presentation is nonspecific. Very recently, a new classification of Intraocular Tuberculosis based on clinical signs, ocular and systemic investigations and therapeutic tests has been published [6]. However, no comparative study has been performed to ascertain its conclusions, and its validation is still missing.

The purpose of this study is to search for diagnostic criterions for tuberculous uveitis. We compared a tuberculous uveitis cohort to other non-tuberculous granulomatous uveitis (mainly sarcoidosis and syphilis, which are the main differential diagnosis). 


\section{Methods}

Patient's data referred to the internal medicine or ophthalmology departments of Nice University Hospital between 2004 and 2014, with a diagnosis of granulomatous uveitis were reviewed. This retrospective observational study was approved by the Ethics committee of Nice University Hospital and, shares the tenets of the declaration of Helsinki.

Patients with diagnosed tuberculous uveitis were included and compared to patients followed-up for a syphilitic or sarcoid uveitis.

The syphilitic uveitis cases were determined as patients with uveitis associated with positive syphilis serology. All the cases healed after appropriate antibiotic therapy (penicillin G or Cephalosporin of third generation once a week or once a day for 3 weeks respectively for a secondary syphilis or a neurosyphilis).

The diagnosis of sarcoid uveitis was considered in patients with biopsy-proven sarcoidosis or in patients presenting with ocular signs consistent with intraocular sarcoidosis and exclusion of other possible differential diagnoses. Patients were treated with systemic corticosteroids alone or in combination with immunosuppressive agents (methotrexate or cyclophosphamide).

The diagnosis of intraocular tuberculosis was considered following the criteria proposed by Graham et al [7].

Suspected cases of intraocular tuberculosis were treated with antituberculous antibiotic: quad-antibiotic therapy (rifampin, isoniazid, pyrazinamide, ethambutol) for tuberculosis disease or a tri-antibiotic therapy (rifampin, isoniazid, pyrazinamide) for latent tuberculosis, during at least two months; then a biantibiotic therapy (rifampin, isoniazid) during 4 to 10 months, associated with a local corticosteroids therapy or systemic topical steroids when needed.

For each patient the following data were collected:

- Epidemiological data: age, sex, ethnicity, travel to countries of endemic tuberculosis, history of exposure to tuberculosis, history of BCG, existence of HIV seropositivity.

- Description of ocular manifestations: lateralization of uveitis, previous localisation, intermediate, posterior or panuveitis, recurrent character, hypertensive uveitis, serpiginous choroiditis, choroidal tubercles and existence of a secondary post-inflammatory cataract. 
- Signs of systemic disease: fever, asthenia, anorexia, weight loss, extrapulmonary (rheumatological, neurological or skin) and pulmonary signs.

- Biological profile (blood formula, blood ionogram, hepatic results and plasma proteins electrophoresis), Human Immunodeficiency Virus (HIV), hepatitis B and C (HBV, HVC), syphilis, toxoplasmosis, herpes simplex virus, rickettsia conorii, toxocariasis and bartonellosis status, angiotensin-converting enzyme ACE, HLA typing.

- Immunological testing: tuberculin skin test, IGRA test : QuantiFERON-TB Gold In- Tube (QFT; Cellestis, Carnegie, VIC, Australia)

- Chest imaging: standard X-ray or computed thoracic tomography with search for the following findings: lymphadenopathy, calcification, interstitial syndrome, nodule or excavation

- Bacteriology: Bacilli Acid-Alcohol-Resistant in expectorations, broncho-alveolar lavage, lumbar puncture or anterior chamber paracentesis.

- Histology if performed: presence of an epithelioid granuloma.

The patients included in the tuberculous uveitis group (TBU) were compared to those included in the syphilitic uveitis group (SYU) and the sarcoid uveitis group (SAU) for each collected data. The variables were compared by one-dimensional and multidimensional analysis with Chi2 test and Fisher's exact test.

\section{Results}

\section{Patient characteristics:}

Eleven syphilitic uveitis, 13 sarcoid uveitis and 22 tuberculous uveitis were included in the study. All had been diagnosed between 2004 and 2014 (Table 1).

The syphilitic uveitis group comprised $81 \%(9 / 11)$ of men with a mean age of 59 years (44 -79). Eighteen per cent (2/11) of the patients came from North Africa and $28 \%(3 / 11)$ had travelled into endemic area of tuberculosis. Eighteen per cent (2/11) were HIV-positive.

The sarcoid uveitis group included $61 \%$ (8/13) of women with a mean age of 40.5 years (23-58). Twenty-three per cent (3/13) of the patients came from North Africa, 7\% (1/13) from Africa, and 38\% (5/13) had travelled to South East Asia. There was no HIV-positive patient.

The tuberculous uveitis group comprised 54\% (12/22) of male with a mean age of 57.9 years (22-91). Seventy-one per cent (15/21) of the patients came from a tuberculosis-endemic region and 90\% (19/22) 
had travelled to tuberculosis-endemic areas. One patient presented with tuberculous uveitis after intravesical BCG therapy. In this group only one patient was HIV-positive.

\section{Clinical ophthalmic presentation (Table 2)}

In the syphilitic uveitis group, $4(40 \%)$ bilateral uveitis, $6(60 \%)$ pan uveitis, and $2(20 \%)$ posterior uveitis were disclosed. Two (20\%) cases of uveitis were recurrent uveitis. Two patients had secondary cataracts and $1(10 \%)$ hypertensive uveitis. There were no choroidal nodule neither serpiginous choroiditis.

In the sarcoid uveitis group, there were $10(77 \%)$ bilateral uveitis rather intermediate (6 (46\%) versus $3(23 \%)$ anterior and $5(38 \%)$ posterior uveitis. Nine $(69 \%)$ uveitis were recurrent ones. No hypertensive uveitis was found. Two patients $(15 \%)$ presented with a secondary cataract. There were no nodule and no serpiginous choroiditis.

In the tuberculous uveitis group, there were $12(55 \%)$ bilateral uveitis, $10(45 \%)$ posterior, $12(55 \%)$ relapsed, and 3 (14\%) hypertensive uveitis. Eight (36\%) patients had secondary cataract, $2(9.1 \%)$ serpiginous choroiditis and $1(4.5 \%)$ choroidal nodules.

\section{Clinical systemic presentation (Table 3 )}

$59 \%(13 / 22)$ patients of the tuberculous uveitis group did not show any systemic signs, while two thirds of the SYU group patients had an evocative clinical presentation of syphilis, such as a syphilitic chancre, a morbilliform skin rash or peripheral lymphadenopathy. 54\% (7/13) of SAU group patients demonstrated clinical manifestations of sarcoidosis, such as erythema nodosum or polyarthralgias.

Biological results:

In the TBU group, 93\% (15/16) of patients had a positive QuantiFERON® and, 86\% (7/8) a positive Mantoux skin-test versus $10 \%(1 / 10)$ and $50 \%(1 / 2)$ respectively in the UNT group. The QuantiFERON® test was not performed in patients included before 2006. Liver function tests showed cholestasis in $22.7 \%(5 / 22)$ of TBU patients and none in the 2 other groups. $12.5 \%(2 / 16)$ of TBU group patients had a positive angiotensin converting enzyme (ACE) versus $60 \%(9 / 15)$ of the UNT group patients (Table 4).

\section{CT scan images:}


In the TBU group, $81 \%(17 / 21)$ of patients had abnormal CT scan with presence of lymphadenopathy, lung nodules or excavations. In the sarcoidosis group, $60 \%(6 / 10)$ of patients disclosed a mediastinal lymphadenopathy but any on them presented with a parenchymal nodule or excavation. (Table 4)

\section{Bacteriology and histology}

Six patients of the TBU group and SAU groups had salivary glands biopsy. Mediastinal, lung or liver biopsies were performed in 5 patients of the TBU group and 6 patients of the SAU group (Table 4). Two patients of the TBU group had an anterior chamber puncture with PCR analysis for Mycobacterium tuberculosis. A lumbar puncture was performed in 9 patients of the SYU group and 2 patients of the TBU group (Table 4). Three cases of lymphocytic meningitis were found in the SYU group and 1 in the TBU group.

To conclude, in univariate analysis, the TBU group was older than the UNT group $(p=0,0079))$. The patients came from a tuberculosis endemic region in $71 \%$ against $25 \%$ in the UNT group ( $\mathrm{p}=0,0002$ ). Ocular syphilis was associated with cataract $(p=0,03)$. Biological cholestasis was present in $22 \%$ in the TBU group versus $0 \%$ in the UNT group $(p=0,036)$. The TBU group disclosed negative ACE ( $p=0,0091)$ and positive Quantiferon $(p<0,0001)$. On CT scan, a nodule was found in $47,47 \%$ of cases in the TBU group versus $6,67 \%$ in the UNT group $(\mathrm{p}=0,0112)$.

\section{Discussion}

The certainty of tuberculous uveitis diagnosis remains impossible in most of cases. There is still no consensus on the diagnostic criteria to adopt. Most doctors use different case definitions of intraocular tuberculosis and systemic investigations or treatments differ among the world [8; 9]. The lack of diagnostic criteria and guidelines for systemic or laboratory investigations in intraocular tuberculosis may be responsible for underdiagnosis or overdiagnosis of tuberculous infections. Therefore, it seems essential to propose diagnostic criteria for tuberculous uveitis. In our study, we found that statistically significant criteria for tuberculous uveitis (Table 5) were age, exposure to tuberculosis in a tuberculousendemic area, negativity of the angiotensin-converting enzyme, positive IGRA test and presence of a lung nodule or excavation on CT scan. Our patients have indeed a mean age of 57.9 years, older than those with non-tuberculous uveitis $(\mathrm{p}=0.0079)$. These data are not in keeping with the literature. Manousaridis K. et al. [10] in Newcastle found a mean age of intraocular tuberculosis patients of 46 
years, whereas Sanghi C. et al. [11] in Manchester, found a mean age of 36 years. This may be explained by the fact that our patients are older on the French Riviera.

Gender does not appear as a diagnostic criterion, which is in accordance with the data of 2 previous English series, which respectively counted $71 \%$ and $41 \%$ of men $[10,11]$ in intraocular tuberculosis patients.

The exposure to a tuberculosis-endemic area appeared to be a highly significant criterion of tuberculous uveitis: $90 \%$ of our tuberculous patients were concerned, while it concerned only $38 \%$ of patients with sarcoid uveitis and $28 \%$ of syphilitic patients $(\mathrm{p}<0.0001)$. Many of our patients came from North Africa $(36 \%)$ and Black Africa (23\%). In the Newcastle case series, patients came from Asia in 52\% and Africa in $10 \%$. In the Manchester series, $55 \%$ of patients were Asian and $18 \%$ African [10, 11]. A predominantly North African and African immigration in France, and Indian and Pakistan in England explain these data.

In our study, we observed that, despite one HIV-seropositive patient, and three patients receiving immunosuppressive drugs (cyclosporin for Behçet's disease and sclerosis, etanercept for ankylosing spondylitis), the patients with tuberculous uveitis were not more immunodepressive than patients from the other groups, as it was observed in the cohort of Newcastle [11].

Although we describe $59 \%$ of bilateral uveitis and $45 \%$ posterior uveitis in our tuberculous uveitis group, the clinical presentation was not specific. This confirms the controversial published data: ophthalmic clinical presentation varies according to the studies. Even if Gupta et al. [12] in 2010 described the presence of posterior synechiae, retinal vasculitis or serpiginous choroiditis in favour of tuberculous uveitis, these elements were rarely present in the clinical description of our patients. The relapsing character of the uveitis and the association with cataract were the only signs in ophthalmic presentation that statistically accounted for the diagnosis of intraocular tuberculosis $(\mathrm{p}=0.0897$ and $\mathrm{p}=$ 0,0326 respectively). It can be explained by the likelihood of a chronic inflammation in tuberculous uveitis, promoting the occurrence of post-inflammatory cataract. The older age of patients with TBU may however be a bias and explain the number of cataract cases in this group.

In our study, tuberculous patients did not show more systemic signs or pulmonary signs than patients from the two other groups, probably as tuberculosis uveitis is mainly related to latent tuberculosis. In the Newcastle cohort, only 2 patients with active tuberculosis demonstrated systemic signs [11]. 
In our study, lack of the angiotensin converting enzyme (ACE) elevation appeared to be a significant diagnostic criterion. Indeed, in the tuberculous group, 2 patients had a positive ACE associated with granulomatous lymphadenitis for one patient and lymphocytic meningitis but the other. Among the 8 patients from the sarcoid uveitis group with positive ACE, 6 demonstrated a granuloma on histology. One patient in the syphilitic uveitis group had a positive ACE but no histologic biopsy was performed. ACE is produced by the macrophages and epithelioid cells present in granulomas and may be a marker for sarcoidosis but also tuberculosis according to some authors [13, 14 and 15]. However, as published in the literature, we ACE levels are higher in sarcoid cases [16, 17].

In our study, we found that cholestasis was frequently observed in patients of TBU group (22.7\% vs. $0 \%, \mathrm{p}=0.0036)$. We are unable to explain this finding.

The IGRA test (QuantiFERON®) is of major help for tuberculous diagnosis $[18,19,20]$. In our cohort, only one patient reaches tuberculous uveitis diagnosis with a negative QuantiFERON®. This patient presented with a pulmonary nodule on the CT scan. He also had a myelodysplastic syndrome, causing chronic lymphopenia, making the IGRA test non-contributory. A patient of non-tuberculosis uveitis group had a positive QuantiFERON®. This patient was initially treated with antitubercular therapy. But due to the absence of good clinical response, the patient was considered to have sarcoidosis and was successfully treated with systemic corticosteroids and immunosuppressive agents (cyclophosphamide and methotrexate). With regard to the tuberculin skin test, statistical analysis could not be performed because of the high number of missing data. As Ball PM showed [21] in 2010, the QuantiFERON® has a positive predictive value greater than the tuberculin skin test in the diagnosis of chronic uveitis. It may be appropriate to perform the IGRA test alone. QuantiFERON® TB Gold In- test is measured by ELISA method: the amount of interferon-gamma secreted by lymphocytes $\mathrm{T}$ gathered in $1 \mathrm{ml}$ of whole blood, after stimulation in vitro by antigens ESAT-6, CFP- 10 and TB7.7 (synthetic peptides representing proteins of Mycobacterium tuberculosis). This test is less sensitive but highly specific as the antigen used in the IGRA test is not found in BCG or any environmental mycobacteria [1]. However, the IGRA does not allow dating the infection or distinguishing between active or latent tuberculosis.

The CT scan was useful in our study, as stated by Ganesh SK. et al. [22] in 2011. CT scan was abnormal in $81 \%$ of cases of their series with probable intraocular tuberculosis. Our study found the same percentage of abnormalities (17 patients out of 22). The presence of a lung nodule was the most 
specific finding accounting for the diagnosis of tuberculosis $(47.6 \%$ vs. $6.67 \%, p=0.0112)$. Indeed, mediastinal lymphadenopathy is also present in sarcoidosis.

The tuberculosis PCR test performed in less than $10 \%$ of cases by anterior chamber puncture was not useful due to its lack of sensibility.

Our study has several limitations. It is retrospective. The family history, exposure to tuberculosis and the history of vaccination with BCG could not be documented in all cases. The number of patients included is limited, but this is the case of almost all the studies dealing with intraocular tuberculosis. Only cohort studies on tuberculin skin test and IGRA test are more powerful [19, 21, 24, 25, 26, 27].

In our study, the response to antitubercular treatment validated our diagnosis. Four patients received quad-antibiotherapy and 18 patients received tri-antibiotherapy, which were associated with corticosteroids systemic therapy for 7 patients and with topical corticosteroid therapy for 11 patients. The response to the treatment was assessed at months 2. The first treatment was switched to isoniazid and rifampin therapies, maintained during 4 to 10 months. Sixteen patients encountered improvement or stabilization of their uveitis at months 2 . All of them were cured at the end of the study.

\section{Conclusion}

Our work found that history of exposure to tuberculosis-endemic area, positive IGRA test and presence of a lung nodule on CT scan, are highly evocative criteria for tuberculous uveitis. We also learned that with older age, negativity of the ACE and possible presence of cholestasis may be to consider for the diagnosis of tuberculous uveitis. 


\section{References}

1. Brézin A. Les uvéites. Société française d'ophtalmologie. France: Masson ; 2010. 760.

2. Bourcier T. Les infections oculaires. Rapport annuel des sociétés d'ophtalmologie de France : Bsof ; 2010. 432.

3. Bodaghi B. Uvéite. Elsevier Eds : France ; 2009. 394.

4. Vasconcelos-Santos, Manfred Zierhut, Narsing A, Rao. Strengths and Weaknesses of Diagnostic Tools for Tuberculous. Ocul Immunol Inflamm. 2009 ; 17(5): 351-355

5. Gupta V, Shougy SS, Mahajan S, Khairallah M, Rosenbaum JT, Curi A, et al. Clinics of ocular Tuberculosis. Ocul Immunol Inflamm. 2015; 23 (1): 14-24

6. Gupta A, Sharma A, Bansal R, Sharma K. Classification of intraocular Tuberculosis. Ocul Immunol Inflamm. $2015 ; 23(1): 7-13$

7. Graham SM, Ahmed T, Amanullah F. Evaluation of tuberculosis diagnostics in children, 1: proposed clinical case definitions for classification of intrathoracic tubercu- losis disease. Consensus from an Expert Panel. J Infect Dis. 2012; 205:S199-S208.

8. Lou SM, Montgomery PA, Larkin KL, Winthrop K, Zierhut M, Rosenbaum J, and members of the Uveitis Specialists Study Group. Diagnosis and Treatment for Ocular Tuberculosis among Uveitis Specialists: The International Perspective. Ocul Immunol Inflamm. 2015; 23(1): 32-39

9. Lou SM, Larkin KL, Winthrop K, Rosenbaum JT, members of Uveitis Specialists Study Group. Lack of Consensus in the Diagnosis and Treatment for Ocular Tuberculosis among Uveitis Specialists. Ocul Immunol Inflamm. 2014 Jul 14; 1-7

10. Manousaridis K, Ong E, Stenton C, Gupta R, Browning AC, Pandit R. Clinical presentation, treatment, and outcomes in presumed intraocular tuberculosis: experience from Newcastle upon Tyne, UK. Eye (Lond). 2013 Apr; 27(4):480-486.

11. Sanghvi C, Bell C, Woodhead M, Hardy C, Jones N. Presumed tuberculous uveitis: diagnosis, management, and outcome. Eye (Lond). 2011 Apr; 25(4):475-480

12. Gupta A, Bansal R, Gupta V, Sharma A, Bambery P. Ocular signs predictive of tubercular uveitis. Am J Ophthalmol. 2010 Apr; 149(4):562-570

13. Fernández Jorge MA, Alonso Mallo E. Angiotensin-converting enzyme (ACE) in sarcoidosis, tuberculosis, silicosis, and coal mining workers. An Med Interna. 1994 Dec;11(12):588-90 
14. Khan AH, Ghani F, Khan A, Khan MA, Khurshid M. Role of serum angiotensin converting enzyme in sarcoidosis. J Pak Med Assoc. 1998 May;48(5):131-3

15. Safianowska A, Grubek-Jaworska H, Droszcz P, Rybus L, Dabrowski A, Zwolska Z et al. Levels of CEA, antibodies against mycobacterial antigens and ACE activity in serum and in BALF in patients with sarcoidosis, tuberculosis and lung cancer--preliminary results. Pneumonol Alergol Pol. 2001; 69(1$2): 5-13$

16. Sainani GS, Mahbubani V, Trikannad V. Serum angiotensin converting enzyme activity in sarcoidosis and pulmonary tuberculosis. J Assoc Physicians India. 1996 Jan ;44(1):29-30

17. Zhang Y, Li X, Wu Z, Fan H. Association between ACE I/D polymorphism and pulmonary tuberculosis in Chinese population. Mol Biol Rep. 2014 May;41(5):3187

18. Lino A, Lalande V, Guetta A, Lebeau B, Heron E, Chouaid C. Diagnosis of tuberculous uveitis: clinical application of an interferon-gamma release assay. Rev Mal Respir. 2011 March;28(3):317-321

19. Modorati G, Berchicci L, Miserocchi E, Scarpellini P, Mantegani P, Bandello F, et al. Clinical application of an in-house ELISPOT assay in patients with suspicious tuberculous uveitis and no signs of active tuberculosis. Eur J Ophthalmol. 2012 Oct; 22(5):808-813

20. Sudharshan S, Ganesh SK, Balu G, Mahalakshmi B, Therese LK, Madhavan HN, et al. Utility of QuantiFERON®-TB Gold test in diagnosis and management of suspected tubercular uveitis in India. Int Ophthalmol. 2012 June; 32(3):217-223

21. Ball P-M, Pernollet M, Bouillet L, Maurin M, Pavese P, Quesada J-L, et al. Usefulness of an in-vitro tuberculosis interferon- $\&$ release assay (T-SPOT.TB) in the first-line check-up of uveitis patients. Ann Med. 2010 Oct; 42(7):546-554

22. Ganesh SK, Roopleen null, Biswas J, Veena N. Role of high-resolution computerized tomography (HRCT) of the chest in granulomatous uveitis: a tertiary uveitis clinic experience from India. Ocul Immunol Inflamm. 2011 Feb; 19(1):51-57

23. Campos WR, Henriques JF, Kritski AL, Curi A, Pimentel RT, Spindola de Miranda S. Tuberculous uveitis at a referral center in southeastern Brazil. J Bras Pneumol. 2008 Feb; 34(2):98-102

24. Ang M, Htoon HM, Chee S-P. Diagnosis of tuberculous uveitis: clinical application of an interferongamma release assay. Ophthalmology. $2009 \mathrm{Jul} ; 116(7): 1391-1396$

25. Ang M, Wong W, Ngan CCL, Chee S-P. Interferon-gamma release assay as a diagnostic test for tuberculosis-associated uveitis. Eye (Lond). 2012 May; 26(5):658-665 
26. Gineys R, Bodaghi B, Carcelain G, Cassoux N, Boutin LTH, Amoura Z, et al. QuantiFERON-TB gold cut-off value: implications for the management of tuberculosis-related ocular inflammation. Am J Ophthalmol. 2011 Sept; 152(3):433-440

27. Hong BK, Khanamiri HN, Bababeygy SR, Rao NA. The utility of routine tuberculosis screening in county hospital patients with uveitis. Br J Ophthalmol. 2014 Aug; 98(8):1091-5 


\section{Legends}

Table 1: Demographic characteristics of patients

W: woman, M: man, age (in years), NA: data non available, EA: tuberculous-endemic area, TBU: tuberculous uveitis, SAU: sarcoid uveitis, SYU: syphilitic uveitis, BCG: Koch bacillus vaccine, HIV: human immunodeficiency virus

\section{Table 2: Ophthalmic presentation}

unilat: unilateral, bilat: bilateral, ant: anterior, int: intermediary, post: posterior, pan: panuveitis, rec: recurrent, HTA: hypertensive uveitis, nodulus: Bouchut tuberculoma, NA : data non available, TBU: tuberculous uveitis, SYU: syphilitic uveitis, SAU: sarcoid uveitis, - : not present sign, +: present sign Table 3: Systemic presentation.

TBU: tuberculous uveitis, SYU: syphilitic uveitis, SAU: sarcoidosis uveitis - : not present sign, +: present sign

\section{Table 4: Paraclinic presentation}

ACE: Angiotensine Converting Enzyme, ADP: mediastinal adenopathy, PCR on PCA: Polymerase Chain Reaction on anterior chamber puncture, CSF: Cerebrospinal Fluid by lumbar puncture

Table 5: Diagnostic criteria of tuberculous uveitis

TBU: tuberculous uveitis, UNT: non tuberculous uveitis, ACE: angiotensin converting enzyme, ICR: intracutaneous tuberculine reaction

\section{Tables}

\begin{tabular}{|c|c|c|c|c|c|c|}
\hline $\mathbf{N}$ & Age (years) & Sex & Ethnic group & Trip to EA & BCG & HIV \\
\hline TBU & $\begin{array}{l}57,9 \\
(22-91)\end{array}$ & $12(54 \%) \mathrm{M}$ & $\begin{array}{l}15(71 \%) \text { origin EA } \\
8(36 \%) \text { Africa } \\
5(23 \%) \text { Maghreb } \\
2(9 \%) \text { Asia }\end{array}$ & $19(90 \%)$ & $1(4 \%)$ & $1(4 \%)$ \\
\hline 1 & 85 & $\mathrm{~W}$ & Cape Verde & yes & NA & NA \\
\hline 2 & 50 & $\mathrm{M}$ & Mali & yes & no & yes \\
\hline 3 & 91 & $\mathrm{M}$ & France & no & Post-BCG intra-bladder & no \\
\hline 4 & 33 & $\mathrm{M}$ & Morocco & yes & NA & no \\
\hline 5 & 61 & $\mathrm{M}$ & Senegal & yes & yes & no \\
\hline 6 & 86 & $\mathrm{M}$ & ND & ND & NA & no \\
\hline
\end{tabular}




\begin{tabular}{|c|c|c|c|c|c|c|}
\hline 7 & 77 & $\mathrm{M}$ & Morocco & yes & NA & no \\
\hline 8 & 80 & $\mathrm{M}$ & France & ND & NA & no \\
\hline 9 & 53 & $\mathrm{~W}$ & Morocco & yes & NA & no \\
\hline 10 & 47 & $\mathrm{~W}$ & Vietnam & yes & NA & no \\
\hline 11 & 85 & $\mathrm{~W}$ & Madagascar & yes & NA & no \\
\hline 12 & 41 & $\mathrm{~W}$ & Cameroun & yes & NA & no \\
\hline 13 & 55 & $\mathrm{~W}$ & France & yes & NA & no \\
\hline 14 & 33 & $\mathrm{M}$ & Senegal & yes & NA & no \\
\hline 15 & 39 & W & India & yes & NA & no \\
\hline 16 & 35 & $\mathrm{M}$ & Côte d'Ivoire & yes & NA & no \\
\hline 17 & 32 & $\mathrm{~W}$ & Burkina & yes & NA & no \\
\hline 18 & 87 & $\mathrm{~W}$ & France & yes & NA & no \\
\hline 19 & 22 & $\mathrm{M}$ & France & yes & NA & no \\
\hline 20 & 74 & $\mathrm{M}$ & Morocco & yes & NA & no \\
\hline 21 & 63 & $\mathrm{~W}$ & France & yes & no & no \\
\hline 22 & 46 & $\mathrm{M}$ & Morocco & yes & no & no \\
\hline SAU & 40,5 & $8 \mathrm{~F}(61 \%)$ & $\begin{array}{l}3(23 \%) \text { Maghreb } \\
1(7 \%) \text { Africa }\end{array}$ & $5(38 \%)$ & NA & 0 \\
\hline 1 & 58 & $\mathrm{~W}$ & France & no & NA & no \\
\hline 2 & 28 & $\mathrm{~W}$ & France & or & NA & no \\
\hline 3 & 38 & $\mathrm{~W}$ & France & no & NA & NA \\
\hline 4 & 23 & $\mathrm{~W}$ & Morocco & yes & NA & no \\
\hline 5 & 39 & $\mathrm{M}$ & France & no & NA & no \\
\hline 6 & 53 & $\mathrm{~W}$ & Cote d'Ivoire & yes & NA & no \\
\hline 7 & 25 & $\mathrm{~W}$ & France & no & NA & no \\
\hline 8 & 35 & $\mathrm{M}$ & Morocco & yes & NA & no \\
\hline 9 & 47 & $\mathrm{~W}$ & Kurdish & no & NA & no \\
\hline 10 & 40 & $\mathrm{M}$ & Morocco & yes & NA & no \\
\hline 11 & 53 & $\mathrm{~W}$ & France & no & NA & NA \\
\hline 12 & 44 & $\mathrm{~W}$ & France & no & NA & no \\
\hline 13 & 43 & $\mathrm{M}$ & France & no & NA & NA \\
\hline SYU & $59(44-79)$ & $9 \mathrm{H}(81 \%)$ & $\begin{array}{l}2(18 \%) \\
\text { Maghreb }\end{array}$ & $\begin{array}{l}3(28 \%) \\
\text { Africa }\end{array}$ & NA & $2(18 \%)$ \\
\hline 1 & 55 & $\mathrm{~W}$ & Algeria & yes & NA & no \\
\hline 2 & 53 & $\mathrm{M}$ & France & no & NA & no \\
\hline 3 & 79 & $\mathrm{M}$ & France & yes, & NA & no \\
\hline 4 & 44 & $\mathrm{M}$ & France & no & NA & yes \\
\hline 5 & 48 & $\mathrm{M}$ & France & no & NA & yes \\
\hline 6 & 44 & $\mathrm{M}$ & France & yes, & NA & no \\
\hline 7 & 54 & $\mathrm{M}$ & France & no & NA & no \\
\hline 8 & 45 & $\mathrm{M}$ & Cape Verde & no & NA & no \\
\hline 9 & 57 & $\mathrm{~W}$ & France & no & NA & no \\
\hline 10 & 60 & $\mathrm{M}$ & France & no & NA & no \\
\hline 11 & 50 & $\mathrm{M}$ & Senegal & yes & NA & no \\
\hline
\end{tabular}

Table 1: Demographic characteristics of patients

W: woman, M: man, age (in years), NA: data non available, EA: tuberculous-endemic area, TBU: tuberculous uveitis, SAU: sarcoid uveitis, SYU: syphilitic uveitis, BCG: Koch bacillus vaccine, HIV: human immunodeficiency virus 


\begin{tabular}{|c|c|c|c|c|c|c|c|c|c|c|c|c|}
\hline $\mathrm{N}$ & Uveitis & & & & & & & & & Choroiditis & Nodules & Cataract \\
\hline & no & yes & & & & & & & & & & \\
\hline & & unila & bilat & ant & int & post & pan & rec & HTA & & & \\
\hline TBU & 0 & $9 / 22$ & 13 & 5 & 1 & 9 & 6 & $12 / 16$ & $3 / 14$ & $2 / 13$ & $1 / 13$ & $8 / 15$ \\
\hline 1 & - & - & + & + & - & - & - & + & + & - & - & + \\
\hline 2 & - & - & + & - & - & - & + & + & - & - & - & + \\
\hline 3 & - & - & + & - & - & - & + & + & - & + & - & - \\
\hline 4 & - & - & + & - & - & + & - & NA & NA & NA & NA & NA \\
\hline 5 & - & + & - & - & + & - & - & + & - & NA & NA & + \\
\hline 6 & - & + & - & - & - & - & + & + & - & - & - & + \\
\hline 7 & - & + & - & + & $\mathrm{N}$ & NA & NA & + & + & NA & ND & NA \\
\hline 8 & - & - & + & - & - & + & - & NA & NA & NA & + & NA \\
\hline 9 & - & - & + & - & - & + & - & + & NA & NA & NA & NA \\
\hline 10 & - & - & + & - & - & - & + & + & ND & NA & NA & + \\
\hline 11 & - & + & - & - & - & + & - & - & - & - & - & - \\
\hline 12 & - & + & - & - & - & + & - & NA & NA & + & NA & NA \\
\hline 13 & - & - & + & + & - & - & - & + & + & - & - & + \\
\hline 14 & - & + & - & + & - & - & - & - & - & - & - & - \\
\hline 15 & - & - & + & - & - & - & + & NA & NA & NA & NA & NA \\
\hline 16 & 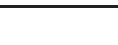 & + & - & NA & $\mathrm{N}$ & NA & NA & - & - & - & - & - \\
\hline 17 & - & - & + & + & - & - & - & NA & NA & NA & NA & NA \\
\hline 18 & - & - & + & - & - & - & + & NA & NA & NA & NA & + \\
\hline 19 & - & - & + & - & - & + & - & + & - & - & - & - \\
\hline 20 & - & + & - & - & - & + & - & + & - & - & - & + \\
\hline 21 & - & - & + & - & - & + & - & + & - & - & - & - \\
\hline 22 & - & + & - & - & - & + & - & - & - & - & - & - \\
\hline USa & 0 & $3 / 13$ & $10 / 1$ & $3 / 13$ & $6 / 1$ & $5 / 1$ & $2 / 13$ & $9 / 13$ & 0 & 0 & 0 & $2 / 13$ \\
\hline 1 & - & - & + & + & - & - & - & + & - & - & - & - \\
\hline 2 & - & - & + & - & + & + & - & + & - & - & - & - \\
\hline 3 & - & - & + & - & - & - & + & + & - & - & - & + \\
\hline 4 & - & - & + & - & + & - & - & + & - & - & - & - \\
\hline 5 & - & - & + & - & + & - & - & + & - & - & - & - \\
\hline 6 & - & - & + & - & - & + & - & - & - & - & - & - \\
\hline 7 & - & + & - & - & - & + & - & - & - & - & - & - \\
\hline 8 & - & + & - & - & - & - & + & + & - & - & - & + \\
\hline 9 & - & - & + & + & - & + & - & + & - & - & - & - \\
\hline 10 & - & - & + & - & - & - & - & + & - & - & - & - \\
\hline 11 & - & + & - & + & + & - & - & - & - & - & - & - \\
\hline 12 & - & - & + & - & + & - & - & - & - & - & - & - \\
\hline 13 & - & - & + & - & + & + & - & + & - & - & - & - \\
\hline USy & 0 & $3 / 10$ & $4 / 10$ & $2 / 10$ & $0 / 1$ & $2 / 1$ & $6 / 10$ & $2 / 10$ & $1 / 10$ & 0 & 0 & $2 / 10$ \\
\hline 1 & - & - & + & NA & $\mathrm{N}$ & NA & NA & + & - & - & - & - \\
\hline 2 & - & - & - & - & - & - & + & - & - & - & - & - \\
\hline 3 & - & - & + & - & - & - & + & + & - & - & - & + \\
\hline 4 & - & - & + & + & - & + & - & - & - & - & - & - \\
\hline 5 & - & - & - & - & - & - & + & - & - & - & - & - \\
\hline 6 & - & - & + & - & - & - & + & - & - & - & - & - \\
\hline 7 & - & - & - & - & - & - & + & - & + & - & - & - \\
\hline 8 & - & + & - & - & - & - & + & - & - & - & - & + \\
\hline 9 & - & + & - & - & - & + & - & - & - & - & - & - \\
\hline 10 & NA & NA & NA & NA & $\mathrm{N}$ & NA & NA & NA & NA & NA & NA & NA \\
\hline 11 & - & + & - & + & - & - & - & - & - & - & - & - \\
\hline
\end{tabular}

Table 2: Ophthalmic presentation

unilat: unilateral, bilat: bilateral, ant: anterior, int: intermediary, pos: posterior, pan: panuveitis, rec: recurrent, HTA: hypertensive uveitis, nodulus: Bouchut tuberculoma, NA : data non available, TBU: tuberculous uveitis, SYU: syphilitic uveitis, SAU: sarcoid uveitis, - : not present sign, +: present sign 


\begin{tabular}{|c|c|c|c|c|}
\hline $\mathbf{N}$ & Systemic signs & Type & $\begin{array}{c}\text { Pulmonary } \\
\text { signs }\end{array}$ & type \\
\hline TBU & 9/22(41\%) & & $3 / 22(14 \%)$ & \\
\hline 1 & - & - & - & - \\
\hline 2 & - & - & - & - \\
\hline 3 & + & Axillary adenopathy, Fever, arthritis & - & - \\
\hline 4 & - & - & - & - \\
\hline 5 & + & $-10 \mathrm{Kg}$ & - & - \\
\hline 6 & - & - & - & - \\
\hline 7 & + & Axillary adenopathy & + & tussis \\
\hline 8 & + & $-10 \mathrm{Kg}$ & - & - \\
\hline 9 & - & - & - & - \\
\hline 10 & - & - & - & - \\
\hline 11 & - & - & - & - \\
\hline 12 & + & Axillary adenopathy; Necrotic skin lesion & - & - \\
\hline 13 & - & - & - & - \\
\hline 14 & + & Recurrent orchitis & - & - \\
\hline 15 & + & Cephalalgia, hypacusia & + & tussis \\
\hline 16 & - & - & - & - \\
\hline 17 & + & $-4 \mathrm{Kg}$, Bazin disease & - & - \\
\hline 18 & - & - & + & pleurisy \\
\hline 19 & - & - & - & - \\
\hline 20 & - & - & - & - \\
\hline 21 & - & - & - & - \\
\hline 22 & + & $\begin{array}{l}\text { Transudation, cephalalgia } \\
\text { Chronic hypertensive disease }\end{array}$ & - & - \\
\hline SAU & $7 / 13(54 \%)$ & - & - & 13 \\
\hline 1 & - & - & - & - \\
\hline 2 & + & Polyarthralgia, telangiectasia & - & - \\
\hline 3 & - & - & - & - \\
\hline 4 & + & $\begin{array}{l}\text { Paraplegia, neurologic bladder } \\
\text { Medullar and cerebrospinal sarcoidosis }\end{array}$ & - & - \\
\hline 5 & - & - & - & - \\
\hline 6 & - & - & - & - \\
\hline 7 & + & Cicatricial keloid & - & - \\
\hline 8 & + & Encephalomeningitis, rhombencephalitis & - & - \\
\hline 9 & + & Erythema nodosum & - & - \\
\hline 10 & + & Raynaud's disease, arthralgia & - & - \\
\hline 11 & - & - & - & - \\
\hline 12 & - & - & - & - \\
\hline 13 & + & Arthralgia, axillary bilateral adenopathy & - & - \\
\hline SYU & $7 / 11(63 \%)$ & - & 0 & 11 \\
\hline 1 & + & Measles rash & - & - \\
\hline 2 & + & Measles rash, Cervical adenopathy & - & - \\
\hline 3 & + & Lymphocytic meningitis & - & - \\
\hline 4 & + & $\begin{array}{l}\text { AEG, fever, inguinal adenopathy, } \\
\text { Measles rash; Pilary leucoplasia, Chancre }\end{array}$ & - & - \\
\hline 5 & - & - & - & - \\
\hline 6 & + & Measles rash & - & - \\
\hline 7 & + & papulo-roseoliforma eruption & - & - \\
\hline 8 & - & - & - & - \\
\hline 9 & + & Arthralgia; Measles rash & - & - \\
\hline 10 & - & - & - & - \\
\hline 11 & - & - & - & - \\
\hline
\end{tabular}

Table 3: Systemic presentation.

TBU: tuberculous uveitis, SYU: syphilitic uveitis, SAU: sarcoidosis uveitis - : not present sign, +: present sign 


\begin{tabular}{|c|c|c|c|c|}
\hline \multicolumn{2}{|c|}{ Group } & Tuberculosis & Sarcoidosis & Syphilis \\
\hline \multicolumn{2}{|c|}{ Number } & 22 & 13 & 11 \\
\hline \multicolumn{2}{|c|}{ Standard biology } & 5 cholestasis & 2 cytolysis & 2 cytolysis \\
\hline \multicolumn{2}{|c|}{ Polyclonal hypergammaglobulinemia } & 6 & 3 & 4 \\
\hline \multicolumn{2}{|c|}{ HIV } & 1 & 0 & 2 \\
\hline \multicolumn{2}{|l|}{ HBV } & 1 & 1 & 1 \\
\hline \multicolumn{2}{|l|}{$\mathrm{HCV}$} & 0 & 0 & 1 \\
\hline \multicolumn{2}{|l|}{ Syphilis } & 0 & 0 & 11 \\
\hline \multicolumn{2}{|c|}{ Toxoplasmosis } & 9 & 1 & 3 \\
\hline \multicolumn{2}{|c|}{ Rickettsia } & 0 & 0 & 0 \\
\hline \multicolumn{2}{|c|}{ Toxocarosis } & 2 & 0 & 2 \\
\hline \multicolumn{2}{|c|}{$\mathrm{ACE}$} & $2 / 16$ & $8 / 13$ & $1 / 4$ \\
\hline \multicolumn{2}{|c|}{ Human Leucocyte Antigens } & $3 \mathrm{~B} 51$ & $1 \mathrm{~B} 51$ & 0 \\
\hline \multicolumn{2}{|c|}{ Quantiferon } & $15 / 16$ & $1 / 7$ & $0 / 3$ \\
\hline \multicolumn{2}{|c|}{ Tuberculin Intracutaneous Reaction } & $7 / 8$ & 0 & $1 / 2$ \\
\hline \multirow[t]{5}{*}{ Scanner } & Normal & $4 / 21(19 \%)$ & $4 / 10(40 \%)$ & $2 / 4(50 \%)$ \\
\hline & ADP & $11 / 21(52,4 \%)$ & $6 / 10(60 \%)$ & $1 / 4(25 \%)$ \\
\hline & Intercurrent Syndrome & $1 / 21(4,7 \%)$ & $2 / 10(20 \%)$ & $0 / 4$ \\
\hline & Nodulus & $10 / 21(47,6 \%)$ & $0 / 10$ & $1 / 4(25 \%)$ \\
\hline & Calcification & $1 / 21(4,7 \%)$ & $0 / 10$ & $0 / 4$ \\
\hline \multicolumn{2}{|c|}{ Salivary Gland Biopsy } & 5 sialadenitis $/ 6$ & 5 sialadenitis $/ 6$ & 0 \\
\hline \multicolumn{2}{|c|}{$\begin{array}{l}\text { Bronchus biopsy /Mediastinal /hepatic } \\
\text { adenopathy (at least one of those biopsies and } \\
\text { diagnosis or not of a granuloma) }\end{array}$} & $\begin{array}{l}2 \text { necrotic granuloma } \\
\qquad / 5\end{array}$ & $\begin{array}{l}6 \text { non necrotic } \\
\text { granuloma } / 6\end{array}$ & 0 \\
\hline \multicolumn{2}{|c|}{ PCR on PCA } & $1 / 2$ & 0 & 0 \\
\hline \multicolumn{2}{|c|}{ Bronchoclysis } & $0 / 10$ & $0 / 1$ & $0 / 2$ \\
\hline \multicolumn{2}{|l|}{ CSF } & $1 / 2$ & 0 & $\begin{array}{l}\text { 3/9 lymphocytic } \\
\text { meningitis }\end{array}$ \\
\hline
\end{tabular}

Table 4: Paraclinic presentation

ACE: Angiotensine Converting Enzyme, ADP: mediastinal adenopathy, PCR on PCA: Polymerase Chain Reaction on anterior chamber puncture, CSF: Cerebrospinal Fluid by lumbar puncture, HIV: Human Immunodeficient Virus, HBV: hepatitis B, HCV: hepatitis C 


\begin{tabular}{|c|c|c|c|c|}
\hline & & TBU & UNT & p-value \\
\hline \multirow{5}{*}{ Epidemiology } & Age (average) & 57,9 & 46,46 & 0,0079 \\
\hline & Men & $55,5 \%$ & $54,17 \%$ & \multirow[t]{2}{*}{0,9794} \\
\hline & Women & $45,45 \%$ & $45,83 \%$ & \\
\hline & Origin of endemic region & $71 \%$ & $25 \%$ & 0,0002 \\
\hline & Travel to endemic region & $90 \%$ & $47,6 \%$ & $<0,0001$ \\
\hline \multirow{11}{*}{ Clinical ophthalmic presentation } & Unilateral uveitis & $40,9 \%$ & $26,09 \%$ & 0,2917 \\
\hline & Bilateral uveitis & $59,09 \%$ & $60,87 \%$ & 0,9031 \\
\hline & Anterior & $23,81 \%$ & $22,73 \%$ & 1 \\
\hline & Intermediary & $5 \%$ & $27,27 \%$ & 0,0961 \\
\hline & Posterior & $45 \%$ & $31,82 \%$ & 0,3796 \\
\hline & Panuveitis & $30 \%$ & $36,36 \%$ & 0,6622 \\
\hline & Recidivating & $75 \%$ & $47,83 \%$ & 0,0897 \\
\hline & Hypertensive & $21,43 \%$ & $4,35 \%$ & 0,1419 \\
\hline & Choroiditis serpiginous & $15,38 \%$ & 0 & 0,1238 \\
\hline & Bouchut nodule & $7,69 \%$ & 0 & 0,3611 \\
\hline & Associated cataract & $53,33 \%$ & $17,39 \%$ & 0,0326 \\
\hline \multirow[b]{2}{*}{ Clinical systemic presentation } & General signs & $40,91 \%$ & $58,33 \%$ & 0,2377 \\
\hline & Pulmonary signs & $13,64 \%$ & 0 & 0,1014 \\
\hline \multirow{6}{*}{ Biology } & Cholestasis & $22,7 \%$ & 0 & 0,0036 \\
\hline & Hypergammaglobulinemia & $27,27 \%$ & $36,84 \%$ & 0,5114 \\
\hline & HIV & $4,76 \%$ & $9,52 \%$ & 1 \\
\hline & HBV & $4,65 \%$ & $8,70 \%$ & 1 \\
\hline & $\mathrm{HCV}$ & 0 & $4,35 \%$ & 1 \\
\hline & ACE & $12,5 \%$ & $60 \%$ & 0,0091 \\
\hline \multirow{2}{*}{ Diagnostic tests } & Quantiferon $^{\circ}$ & $93,75 \%$ & $10 \%$ & $7,097.10^{\wedge}-5$ \\
\hline & ICR & $87,5 \%$ & $50 \%$ & NR \\
\hline \multirow{4}{*}{ Medical imaging } & Normal scanner & $19,05 \%$ & $40 \%$ & 0,26 \\
\hline & Lymphonodus & $52,38 \%$ & $46,67 \%$ & 1 \\
\hline & Nodule/excavation & $47,6 \%$ & $6,67 \%$ & 0,0112 \\
\hline & Interstitial syndrome & $4,76 \%$ & $13,33 \%$ & 0,5588 \\
\hline
\end{tabular}

Table 5: Diagnostic criteria of tuberculous uveitis

TBU: tuberculous uveitis, UNT: non tuberculous uveitis, ACE: angiotensin converting enzyme, ICR: intracutaneous tuberculine reaction 


\section{Table des Matières}

Remerciements

p.7

I- Introduction

p.11

II- Matériels et Méthodes

p. 13

III- Résultats

p. 16

1. Caractéristiques des patients

p. 16

2. Présentation clinique ophtalmologique

p. 18

3. Présentation clinique systémique

p. 21

4. Résultats biologiques

p. 21

5. L'imagerie

p. 21

6. La bactériologie et 1'histologie

p. 22

IV- Discussion

p. 23

$\mathrm{V}$ - Conclusion

p.29

VI- Résumé

p.31

VII- Bibliographie

p. 32

VIII- Annexes

p.34 